\title{
Analysis of PIV measurements using modal decomposition techniques, POD and DMD, to study flow structures and their dynamics within a stirred-tank reactor
}

\author{
Anne de Lamotte ${ }^{\mathrm{a}, \mathrm{b}}$, Angélique Delafosse ${ }^{\mathrm{a}}$, Sébastien Calvo ${ }^{\mathrm{a}}$, Dominique Toye ${ }^{\mathrm{a}}$ \\ ${ }^{a}$ Department of Chemical Engineering - Products, Environment, Processes (PEPs), University of Liège, Allée du 6 août 17B, 4000 Liège, Belgium \\ ${ }^{b}$ National Funds for Scientific Research (FRS-FNRS), Rue d'Egmont 5, 1000 Brussels, Belgium
}

\begin{abstract}
The present work is a comparative analysis of Proper Orthogonal Decomposition (POD) and Dynamic Mode Decomposition (DMD) computed on experimental turbulent velocity fields measured in a 20L-tank stirred by two Rushton impellers at two rotating speeds, $N=150$ and $300 \mathrm{rpm}$. POD identifies flow structures that optimally capture the total kinetic energy of the flow, while DMD identifies structures that significantly contribute to the dynamics of the flow. The experimental data, i.e. the instantaneous velocity fields $U_{r}(r, z, t)$ and $U_{z}(r, z, t)$, come from 2-D Particle Image Velocimetry (PIV). The flow motion is turbulent, and it occurs over a wide range of length and time scales, from equipment-dependent large-scale coherent structures to the smallest-scale eddies where energy dissipation takes place. It thus provides an interesting benchmark case for the comparison between POD and DMD, which are based on energy and dynamic analysis, respectively. POD analysis reveals that the most energetic structures are related to the inherent periodic unsteadiness due to the relative motion between the rotating impeller blades and the non-moving baffles. Apart from the mean field, the first most energetic group of modes is related to trailing vortices induced by the Rushton turbines and is associated to a frequency equivalent to the blade passage frequency and its overtones. The second most energetic group of modes is related to vortical structures in the impeller stream and is associated to a frequency equivalent to the rotating speed. DMD analysis identifies flow structures that are found similar to these most energetic modes, although differences appear due to the fact that DMD isolates structures associated to a single frequency and their corresponding growth/decay rate. As in POD, the relative importance of each DMD mode can be estimated using an appropriately defined energy criterion. Comparison of the results from both modal decomposition methods points out their complementarity and their potential for describing the spatial and time characteristics of the flow within a stirred tank.
\end{abstract}

Keywords: Stirred tanks, Hydrodynamics, PIV, POD, DMD

\section{Introduction}

Most industrial stirred-tank reactors are operating under turbulent conditions. Their flow is thus characterized by structures of various length and time scales, whose effects on physical phenomena such as mixing and mass transfer potentially depend on the reactor scale.

Turbulent flows are thus ruled by the motion of eddies characterized by a very wide range of length and time scales. The largest ones exhibit discernible and organized structures containing most of the kinetic energy. Their size is comparable to the characteristic dimension of the system (generally one tenth of the impeller diameter $D$ ). These macro swirling motions, dependent on the conditions of turbulence generation (consequently the agitation system), start a cascade of eddies of decreasing size down to the smallest of turbulent eddies (Kolmogorov length scale $\eta$ ) until they are finally dissipated into heat by viscous forces. The smallest eddies within the inertial and viscous ranges correspond to flow structures that are independent of the conditions of turbulence generation. All these flow structures, from the largest to the smallest scale, have to be properly taken into account to understand and model their dynamical behavior. Therefore, flow within mechanically- agitated vessels is quite complex with various time and spatial features. A first step in the analysis of such systems is to look for and extract physically important spatial structures.

Over the years, many studies have been conducted in order to understand the physical mechanisms related to these flow structures and their length and time characteristics. Various data-processing methods as well as experimental and computational techniques have been widely developed and used to this aim. For instance, here is a non-exhaustive list of publications and review papers addressing these fields of research differently, e.g. (i) experimental techniques: Derksen et al. (1999), Mavros (2001), Rammohan et al. (2001), Escudié and Liné (2003), Chiti et al. (2011); (ii) computational techniques: Brucato et al. (1998), Deglon and Meyer (2006), Guha et al. (2006), Jahoda et al. (2007), Joshi et al. (2011); and (iii) processing methods: Montes et al. (1997), Galletti et al. (2003), Roussinova et al. (2004), Moreau and Liné (2006), Joshi et al. (2009).

On the experimental side, Particle Image Velocimetry (PIV) has become a major tool (e.g. Sheng et al. (2000), Sharp and Adrian (2001), Aubin et al. (2004), Gabriele et al. (2009), Delafosse et al. (2011)). It is indeed particularly efficient, providing measurements of flow quantities with a quite high degree 
of spatial and/or time resolution. Furthermore, this experimental method is widely used for numerical model validation (e.g. Sheng et al. (1998), Deen et al. (2002), Delafosse et al. (2008), Zadghaffari et al. (2009), Delafosse et al. (2014)). However, this advanced experimental technique generates a huge amount of detailed data relative to a large volume fraction of the stirred vessel. These results are in many cases neither straightforward nor easy to interpret due to the complexity of the turbulent flow and of the geometry, and to the interactions of different mechanisms.

Mathematical flow decomposition methods, such as Proper Orthogonal Decomposition (POD) and Dynamical Mode Decomposition (DMD), are extremely interesting tools to analyze hydrodynamics within stirred-tanks, providing detailed information about the dominant largest flow structures. These two techniques effectively compress and summarize large amounts of data to extract details about physical phenomena. They both decompose the turbulent flow fields into time and spatial features to provide a simpler representation of the complex timedependent physical mechanism. The POD decomposition leads to the identification of the most energetic spatial structures along with time behavior, while the DMD decomposition leads to the extraction of spatial structures associated to a specific frequency and expansion rate. In other words, POD sorts modes according to their energy content, and not according to their dynamical importance. The POD modes are generally associated with a mix of frequencies. DMD analysis addresses this issue as each DMD mode is associated with a particular frequency. POD is one of the most widely used techniques in quantitatively describing turbulent flow fields, including within stirred-tanks. For the latter, POD has proved useful for the extraction of coherent structures (Tabib and Joshi (2008), Liné et al. (2013)), the detection of transition to turbulence (Raju et al., 2005) and of flow instabilities (Hasal et al. (2000), Hasal et al. (2004), Ducci et al. (2008)). The DMD technique is one of the most recent post-processing tools, giving an enhanced understanding of the system dynamics (e.g. Rowley et al. (2009), Schmid (2010), Schmid (2011)). For instance, Rowley et al. (2009) have applied DMD to 3D jet-in-crossflow numerical data, effectively capturing the dominant frequencies and elucidating the related spatial structures. In Schmid (2010), DMD demonstrations are presented, for experimental and numerical data, consisting of plane channel flow, flow over a two-dimensional cavity, wake flow behind a flexible membrane and a jet passing between two cylinders. For all these examples, the method has proved to be a robust and reliable tool to extract spatio-temporal coherent structures.

Recently, similarities between POD and DMD have been evidenced by several authors (Semeraro et al. (2012), Schmid et al. (2012), Zhang et al. (2014), Sakowitz et al. (2014)). The modes obtained with the two methodologies tend to be similar when the flow behaves as a periodic oscillator. However, DMD has never been applied to stirred-tank flows yet.

The focus of this study is therefore the application of POD and DMD to turbulent velocity fields inside a stirred-tank in order to identify the most dominant flow patterns along with their time behavior. For this purpose, 2D-PIV measurements of the turbulent flow in a dual Ruhston impeller lab-scale baffled stirred-tank reactor have been performed for two rotating speeds. The experimental results are first briefly described in terms of radial and axial mean velocity and rms turbulent velocity fields, i.e. $\overline{U_{r}}(r, z)$ and $\overline{U_{r}}(r, z)$, and $\sqrt{\overline{u_{r}^{\prime 2}}}(r, z)$ and $\sqrt{\overline{u_{z}^{\prime 2}}}(r, z)$. The POD and DMD methods are then applied to the instantaneous velocity fields to extract the most relevant spatial flow structures and their dynamics. For both modal decompositions, results have been compared with original data and further discussed on the basis of the rotating speed of the impeller and of the acquisition parameters of the experimental technique. Although 2D-PIV measurements are not adequately time-resolved, POD and DMD clearly relate the proper time dynamics to the physically relevant modes. These dominant spatial structures identified by both methods are associated, as shown by other works (e.g. Ducci et al. (2008), Liné et al. (2013), Rowley et al. (2009), Semeraro et al. (2012)), to the flow periodicity. For instance, by means of analysis of the whole vertical plane, in the detected organized motions, the vortex shedding, or trailing vortices, generated by the impellers blades are characterized by oscillation frequencies taken for the blade passage frequency and its higher harmonics. Finally, the comparison between both decomposition techniques present the interest of DMD as a characterization tool for mixing tanks.

\section{Material and Methods}

\subsection{Stirred-tank configuration}

The investigated stirred-tank configuration, presented in Fig.(1), has already been used in previous works (Delafosse et al. (2014), de Lamotte et al. (2017)). It consists of a flatbottom cylindrical vessel of diameter $T=0.22 \mathrm{~m}$, made of transparent Plexiglas, with four equally-spaced baffles. Agitation is provided by a dual-impeller system, composed of two four-blade Rushton turbines of diameter $D \approx 0.5 T=0.1 \mathrm{~m}$ with a spacing $2 D$ and placed at an off-bottom clearance $D$, which implies a submergence of the upper impeller of $\sim 1.5 D=0.14$ $\mathrm{m}$. The working fluid is distilled water at $20^{\circ} \mathrm{C}$ (density $\rho_{\mathrm{L}}=$ $998 \mathrm{~kg} \cdot \mathrm{m}^{-3}$, kinematic viscosity $\left.v_{\mathrm{L}}=1.002 \cdot 10^{-6} \mathrm{~m}^{2} \cdot \mathrm{s}^{-1}\right)$. The liquid height-to-diameter ratio is equal to 2 , which corresponds to a working volume $V=16.5 \mathrm{~L}$. The tank is immersed in a water-filled transparent Plexiglas rectangular container in order to minimize optical distortion due to cylindrical vessel geometry. All experiments are performed at two rotating speeds $N=150$ and $300 \mathrm{rpm}$, corresponding to a Reynolds number $R e=2.4 \cdot 10^{4}$ and $4.7 \cdot 10^{4}$ respectively. The overall turbulence dissipation rate is evaluated from measured values of the mechanical torque $M$ exerted on the impeller shaft: $\bar{\varepsilon}=0.05$ \pm 0.001 and $0.45 \pm 0.003 \mathrm{~m}^{2} \cdot \mathrm{s}^{-3}$ for $N=150$ and $300 \mathrm{rpm}$ (Eq. (1)). The corresponding power numbers over the two impellers are equal to $P_{0}=5.3 \pm 0.11$ and $5.9 \pm 0.03$ for $N=150$ and 300 rpm.

$$
\frac{P}{V}=\frac{2 \pi N\left(M-M_{0}\right)}{V}=\rho_{\mathrm{L}} \bar{\varepsilon}=\frac{\rho_{\mathrm{L}} N^{3} D^{5} P_{0}}{V}
$$

This slight difference in total power number suggests that the turbulent regime within the stirred-tank at $N=150 \mathrm{rpm}$ is not 


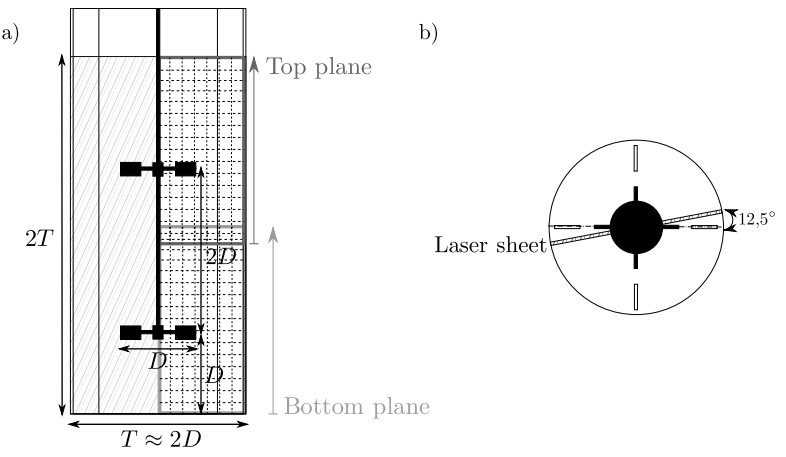

Figure 1: Cross-sectional (a) and top (b) views of the stirred tank configuration

fully developed yet. This observation is discussed in more detail in Sect.3.1. Moreover, it is worth noting that, even for high Reynolds numbers $R e \geq 20000$, power number of Rushton disc turbines, as well as mean flow and turbulence characteristics, are affected by the impeller geometry, i.e. the ratio of impeller blades and disc thickness to impeller or tank diameter (Bujalski et al. (1987), Rutherford et al. (1996)).

\subsection{Local data acquisition methods}

The liquid instantaneous radial and axial velocity fields, $U_{r}(r, z, t)$ and $U_{z}(r, z, t)$, are determined using the PIV technique. The experimental set-up and processing method have already been described in detail previously (de Lamotte et al., 2017).

The measurement system and acquisition procedure are then briefly reported below. The PIV apparatus used in this study is commercialized by Dantec Dynamics (Denmark). It includes a pulsed laser Nd:YAG (Litron, DualPower 65-15, $532 \mathrm{~nm}, 2 \times 65$ $\mathrm{mJ})$, two FlowSense EO cameras $(2048 \times 2048$ pixel, $32 \mathrm{~Hz})$ fitted with a Zeiss lens (Makro-Planar T* 2/50 ZF, $50 \mathrm{~mm}, \mathrm{f} / 2.0$ ) surmounted by a $550 \mathrm{~nm}$ high-pass filter, a high-resolution synchronization unit (Berkeley Nucleonics Corporation, Model 575 Pulse/Delay Generator) and the Dynamic Studio software. Measurements are performed on a vertical plane passing through the impeller axis and placed $12.5^{\circ}$ behind one of the baffles. The image pairs are acquired by both cameras with their optical axis perpendicular to the laser sheet: one for the bottom of the tank and the other one for the top, as depicted in Fig.(1).

The fluid is seeded with inert polyamide particles (density $\rho_{\mathrm{P}}$ $=1030 \mathrm{~kg} \cdot \mathrm{m}^{-3}$, mean diameter $\left.d_{\mathrm{P}}=5 \mu \mathrm{m}\right)$. Their positions are recorded at two distinct time resolutions, $\Delta t=0.0625 \mathrm{~s}$ (16 $\mathrm{Hz}$ ) and $0.0417 \mathrm{~s}(24 \mathrm{~Hz})$. To increase the maximal acquisition speed, a binning $2 \times 2$ has to be performed. Indeed, binning is the procedure of combining the signal from a number of adjacent pixels into an output for a single pixel (grouped into a function unit). As a result, the generated charge carriers accumulate from the single pixels. Consequently, as the binning technique consults the values around, it can be said that it performs local smoothing. The overall number of pixels that need to be read out is reduced, enabling then a significant increase in maximal frame rate $(16 \rightarrow 24 \mathrm{~Hz})$ and in maximal number of acquired images $(725 \rightarrow 2900$ images for each camera). Spatial resolution is then modified, $0.6 \mathrm{~mm}$ or $1.2 \mathrm{~mm}$ (binning $2 \times 2$ ), and the time between the laser pulses is adapted. Moreover, it is worth noting that the signal-to-noise ratio is higher due to reduced read noise contributions and increased signal combinations.

Acquisition frequencies have been limited by the present PIV system; the time step ideally needs to be kept to a minimum to accurately capture the smallest time scales. It is clear that the time interval between two consecutive snapshots can be very important for modal decomposition methods, but the aim of the present work is to assess the capabilities of POD and DMD to detect the dominant flow behavior on the basis of PIV data.

From each image pair, the velocity vectors are computed using a data-processing procedure: (i) Adaptive-PIV treatment (interrogation areas - $64 \times 64$ pixel $^{2} \rightarrow 8 \times 8$ pixel $^{2}$ with a grid step size of 4), (ii) peak validation, (iii) outlier detection, and (iv) range validation (based on the standard deviation of each velocity component). For both rotating speeds, the number of image pairs is selected to assure statistical convergence of the velocity and turbulence quantities: 3 sets of 725 image pairs or 2900 image pairs (binning $2 \times 2$ ).

In a previous work (de Lamotte et al., 2017), the PIV measurements performed at $N=300 \mathrm{rpm}$ have been used to estimate spatial distributions of only two of the three characteristic turbulent length-scales from spatial correlation functions of the velocity: the Taylor macro (or integral) length-scale $\mathcal{L}$ and the Taylor micro length-scale $\ell$. The Taylor micro-scale distribution has been found lying between $0.5 \mathrm{~mm}$ and $5 \mathrm{~mm}$ within the measurement plane with a mean value $\bar{\ell}=2.5 \mathrm{~mm}$; this order of magnitude is coherent with the finer spatial resolution of the PIV technique $(\Delta x=0.6 \mathrm{~mm})$ which is thus small enough to capture the micro-scale $\ell$ and to estimate the kinetic energy $k$. The calculations have been consequently conducted for $N=150 \mathrm{rpm}$, and the results are really close to those for $N=300 \mathrm{rpm}$. The Taylor micro-scale distribution has been found lying between $0.5 \mathrm{~mm}$ and $5.5 \mathrm{~mm}$ within the measurement plane with a mean value $\bar{\ell}=2.65 \mathrm{~mm}$.

However, to accurately deduce the spatial distribution of the Kolmogorov scale $\eta$, the PIV spatial resolution should be equal to or smaller than $\eta$. The mean Kolmogorov scale over the whole vessel $\bar{\eta}$ can be estimated from the global dissipation rate, $\bar{\varepsilon}=0.05$ and $0.45 \mathrm{~m}^{2} \cdot \mathrm{s}^{-3}$ for $N=150$ and $300 \mathrm{rpm}$, using Eq.(2) and is found to be around 70 and $40 \mu \mathrm{m}$. The finer spatial resolution under the present experimental conditions thus corresponds to about 9 and 15 times the mean Kolmogorov scale; the PIV data are definitely filtering the smallest scales of turbulence.

$$
\bar{\eta}=\left(\frac{v^{3}}{\bar{\varepsilon}}\right)^{\frac{1}{4}}
$$

\subsection{Local data processing methods}

The modal decomposition techniques, POD and DMD, are briefly introduced in this section. More details may be found in the literature, e.g. POD by Sirovich (1987), Berkooz et al. (1993), Chatterjee (2000), Liné et al. (2013) and Liné (2016), and DMD by Rowley et al. (2009), Schmid (2010), Schmid (2011), Schmid et al. (2011) and Tissot et al. (2014). 
The main goal is to analyze, in parallel, the spatial structures and their time evolution in a given data set to identify their dominant behaviors.

POD and DMD techniques decompose any data (vector or scalar) ensemble $\psi$ (e.g. the instantaneous velocity fields $\mathbf{U}(r, z, t))$ into temporal amplitudes $\boldsymbol{\theta}(t)$ and spatial modes $\boldsymbol{\phi}(\mathbf{x})$ :

$$
\boldsymbol{\psi}(\mathbf{x}, t)=\sum_{\mathrm{I}=1}^{\mathrm{N}} \boldsymbol{\theta}_{\mathrm{I}}(t) \boldsymbol{\phi}_{\mathrm{I}}(r, z)
$$

The dataset of instantaneous velocity fields $\mathbf{U}(r, z, t)$, obtained by the 2D-PIV experiments with an equal time interval $\Delta t$ between two subsequent fields, are represented in a form of sequence of snapshots, i.e. $\mathrm{N}$ snapshots $U_{r \mathrm{k}}(r, z)$ and $\mathrm{N}$ snapshots $U_{z \mathrm{k}}(r, z)$ where $\mathrm{k}$ is the index of the instantaneous event $(\mathrm{k} \in[1, \mathrm{~N}])$.

To do so, the snapshot sequence needs to be properly written out. The $2 \times \mathrm{N}$ snapshots $\mathbf{U}_{\mathrm{k}}(r, z)$ are assembled column-wise in a $2 \mathrm{RC} \times \mathrm{N}$ snapshot matrix $\mathcal{V}_{1}^{\mathrm{N}}$ :

$$
\mathcal{V}_{1}^{\mathrm{N}}=\left[\begin{array}{lll}
\mathbf{V}_{1} \ldots \mathbf{V}_{\mathrm{k}} \ldots \mathbf{V}_{\mathrm{N}-1} \mathbf{V}_{\mathrm{N}}
\end{array}\right] \text { with } \mathbf{V}_{\mathrm{k}}=\left[\begin{array}{c}
U_{r \mathrm{k}}\left(r_{1}, z_{1}\right) \\
\vdots \\
U_{r \mathrm{k}}\left(r_{\mathrm{R}}, z_{1}\right) \\
U_{r \mathrm{k}}\left(r_{1}, z_{2}\right) \\
\vdots \\
U_{r \mathrm{k}}\left(r_{\mathrm{R}}, z_{\mathrm{C}}\right) \\
U_{z \mathrm{k}}\left(r_{1}, z_{1}\right) \\
\vdots \\
U_{z \mathrm{k}}\left(r_{\mathrm{R}}, z_{1}\right) \\
U_{z \mathrm{k}}\left(r_{1}, z_{2}\right) \\
\vdots \\
U_{z \mathrm{k}}\left(r_{\mathrm{R}}, z_{\mathrm{C}}\right)
\end{array}\right]
$$

where $\mathrm{R}$ and $\mathrm{C}$ are the number of rows and the number of columns in the regular grid of the PIV measurement $(r, z)$-plane, respectively.

Depending on the chosen modal decomposition, different types of information can be obtained on the measured fields, which highlight different physical aspects. The POD decomposition leads to the identification of the most energetic spatial structures along with time behavior, while the DMD decomposition leads to the extraction of spatial structures associated to a specific frequency and expansion rate.

\subsubsection{Proper Orthogonal Decomposition}

The POD technique can be considered as a purely statistical method based on the maximization of the energy over the complete data ensemble. The goal of this technique is the extraction of energetic information, i.e. the identification of the most energetic spatial structures from a given snapshot sequence.

The POD algorithm, whose procedural steps are given in Algorithm 1 in Appendix, is based on determining the set of modes $\phi^{\mathrm{POD}}(r, z)$ that gives the optimal, in a least-square sense, approximation of $\mathbf{U}_{\mathrm{k}}(r, z)$ :

$$
\min _{\phi^{\mathrm{POD}}}\left[\mathbf{U}_{\mathrm{k}}(r, z)-\sum_{\mathrm{I}=1}^{\mathrm{N}} \theta_{\mathrm{kI}}^{\mathrm{POD}} \cdot \boldsymbol{\phi}_{\mathrm{I}}^{\mathrm{POD}}(r, z)\right]^{2}
$$

while fulfilling the orthogonality of the modes:

$$
\left(\phi_{\mathrm{I}}^{\mathrm{POD}}\right)^{\mathrm{T}} \boldsymbol{\phi}_{\mathrm{J}}^{\mathrm{POD}}=\boldsymbol{\delta}_{\mathrm{IJ}}
$$

where $\left(\phi_{\mathrm{I}}^{\mathrm{POD}}\right)^{\mathrm{T}}$ is the transpose of $\boldsymbol{\phi}_{\mathrm{I}}^{\mathrm{POD}}$ and $\delta_{\mathrm{IJ}}$ the Kronecker delta. The minimization problem (Eq.(5)) is equivalent to an eigenvalue problem and can be written in the discrete form (Sirovich (1987)) as:

$$
\mathbf{R} \boldsymbol{\phi}^{\mathrm{POD}}=\lambda^{\mathrm{POD}} \boldsymbol{\phi}^{\mathrm{POD}} \text { where } \mathbf{R}=\frac{1}{\mathrm{~N}} \mathcal{V}_{1}^{\mathrm{N}}\left(\mathcal{V}_{1}^{\mathrm{N}}\right)^{\mathrm{T}}
$$

Due to the symmetry of the cross-correlation matrix $\mathbf{R}$, the eigenvalues $\lambda^{\mathrm{POD}}$ are real and describe the energy content of the spatial structures represented by the eigenvectors $\phi^{\mathrm{POD}}$.

Each snapshot of the flow can be expanded into a series of POD modes (Eq.(3)). However, the time coefficients first need to be known at all the time instants $\mathrm{k}$. They are obtained by projecting each instantaneous velocity field on the modes:

$$
\theta_{\mathrm{I}}^{\mathrm{POD}}(\mathrm{k})=\mathbf{V}_{\mathrm{k}} \phi_{\mathrm{I}}^{\mathrm{POD}}
$$

In other words, this method computes the correlation matrix of the set of interdependent data, subsequently diagonalizes it and thus yields decorrelated orthogonal structures while retaining as much as possible the variation in the original dataset. POD provides a basis sorted with respect to the largest data variance and therefore captures a maximal energy content in each identified structure while simultaneously maintaining a zero correlation between all structures. Considering this description, the decomposition procedure provides a ranking of structures in which the perturbation energy of the flow is optimally captured, instead of reproducing the dynamics of the flow.

\subsubsection{Dynamic Mode Decomposition}

The DMD technique is based on the eigendecomposition of a best-fit linear operator that approximates the dynamics present in the data. The snapshots are assumed to be generated by a linear dynamic system. Nevertheless, flow dynamics are typically non-linear. The meaning of DMD for non-linear system is given by its relationship with the Koopman, or composition, operator (Rowley et al. (2009), Mezic (2013), Tu et al. (2014)). The Koopman operator is a linear but infinite-dimensional operator, that captures the evolution of observables (e.g., of fluid velocity) describing any, even non-linear, dynamical system. DMD analysis can be regarded as a numerical approximation to Koopman spectral analysis, and in this context DMD is applicable to non-linear systems.

The goal of the DMD technique is the extraction of dynamic information, i.e. the identification of spatial structures that oscillate and/or grow/decay at specific rates.

The DMD algorithm, whose procedural steps are given in Algorithm 2 in Appendix, is built on two main assumptions. The first hypothesis is that there exists a linear operator $\mathbf{A}$ to have the snapshots step forward in time:

$$
\mathbf{V}_{\mathrm{k}+1}=\mathbf{A} \mathbf{V}_{\mathrm{k}} \text { for } \mathrm{k}=1, \ldots, \mathrm{N}-1
$$


If the flow fields stem from a non-linear process, this assumption amounts to a linear tangent approximation. In the special case of a purely linear process, no approximation is invoked by assuming a constant mapping. In any case, the snapshot matrix (Eq.(4)) can be formulated as a Krylov sequence (Schmid, 2010):

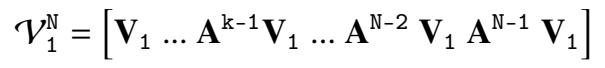

Consequently, the following relationship can be written:

$$
\mathcal{V}_{2}^{\mathrm{N}}=\mathbf{A}\left[\begin{array}{lllll}
\mathbf{V}_{1} & \ldots & \mathbf{V}_{\mathrm{k}} & \ldots & \mathbf{V}_{\mathrm{N}-1}
\end{array}\right]=\mathbf{A} \mathcal{V}_{1}^{\mathrm{N}-1}
$$

Matrix A is considered constant during the time spanned by the snapshots and contains the time information of the dynamic process. The next step is to extract the dynamic characteristics, i.e. to determine the eigenvalues and eigenvectors of $\mathbf{A}$ without determining A itself.

When the number of snapshots of the sequence $\mathcal{V}_{1}^{\mathrm{N}}$ increases $(\mathrm{N}$ large enough), it is reasonable to assume that the vectors $\mathbf{V}_{\mathrm{k}}$ become linearly dependent (Ruhe, 1984). In other words, adding further flow fields to the data sequence is not improving the vector space spanned by $\mathcal{V}_{1}^{\mathrm{N}}$. The second hypothesis is to consider that the vector $\mathbf{V}_{\mathrm{N}}$ can be written as a linear combination of the previous ones as:

$$
\mathbf{V}_{\mathrm{N}}=a_{1} \mathbf{V}_{1}+\ldots+a_{\mathrm{k}} \mathbf{V}_{\mathrm{k}}+\ldots+a_{\mathrm{N}-1} \mathbf{V}_{\mathrm{N}-1}+\mathbf{r}=\mathcal{V}_{1}^{\mathrm{N}-1} \mathbf{a}+\mathbf{r}
$$

where $\mathbf{r}$ is the residual vector and $\mathbf{a}$ is the vector containing the coefficients $a_{\mathrm{k}}$ associated to each $\mathbf{V}_{\mathrm{k}}$. Therefore, by combining the two hypotheses, the snapshot sequence $\mathcal{V}_{2}^{\mathrm{N}}$ (Eq.(11)) becomes (Schmid, 2010):

$$
\begin{aligned}
& \mathbf{A}\left[\begin{array}{lllll}
\mathbf{V}_{1} & \ldots & \mathbf{V}_{\mathrm{k}} & \ldots & \mathbf{V}_{\mathrm{N}-1}
\end{array}\right]=\left[\begin{array}{llllll}
\mathbf{V}_{2} & \ldots & \mathbf{V}_{\mathrm{k}+1} & \ldots & \mathbf{V}_{\mathrm{N}}
\end{array}\right] \\
& =\left[\begin{array}{lllll}
\mathbf{V}_{2} & \ldots & \mathbf{V}_{\mathrm{k}+1} & \ldots & \mathcal{V}_{1}^{\mathrm{N}-1} \mathbf{a}
\end{array}\right]+\mathbf{r e}_{\mathrm{N}-1}^{\mathrm{T}} \\
& \Leftrightarrow \mathbf{A} \mathcal{V}_{1}^{\mathrm{N}-1}=\mathcal{V}_{2}^{\mathrm{N}}=\mathcal{V}_{1}^{\mathrm{N}-1} \mathbf{S}+\mathbf{r e}_{\mathrm{N}-1}^{\mathrm{T}}
\end{aligned}
$$

where $\mathbf{e}_{\mathrm{N}-1}^{\mathrm{T}}$ is the transpose of the $(\mathrm{N}-1)^{\text {th }}$ unit vector. The matrix $\mathbf{S}$ is of Companion type and is uniquely defined by the coefficients $a_{\mathrm{k}}$ :

$$
\mathbf{S}=\left[\begin{array}{ccccc}
0 & & & & a_{1} \\
1 & 0 & & & a_{2} \\
& \ddots & \ddots & & \vdots \\
& & 1 & 0 & a_{\mathrm{N}-2} \\
& & & 1 & a_{\mathrm{N}-1}
\end{array}\right]
$$

The eigenvalues of $\mathbf{S}$ are known to approximate some of the eigenvalues of the full system matrix A (Schmid, 2010). This companion matrix can be computed by a straightforward leastsquare procedure through a $\mathrm{QR}$-decomposition of $\mathcal{V}_{1}^{\mathrm{N}-1}$ :

$$
\mathcal{V}_{2}^{\mathrm{N}} \approx \mathcal{V}_{1}^{\mathrm{N}-1} \mathbf{S}=\mathbf{Q R S} \Leftrightarrow \mathbf{S}=\mathbf{R}^{-1} \mathbf{Q}^{*} \mathcal{V}_{2}^{\mathrm{N}}
$$

where $\mathbf{Q}^{*}$ is the complex conjugate transpose of $\mathbf{Q}$. The dynamic modes $\phi^{\mathrm{DMD}}(r, z)$ and their associated frequencies $f^{\mathrm{DMD}}$ and growth rates $g^{\mathrm{DMD}}$ are then found by:

$$
\begin{aligned}
& \boldsymbol{\phi}_{\mathrm{I}}^{\mathrm{DMD}}=\mathcal{V}_{1}^{\mathrm{N}-1} \mathbf{Y}_{\mathrm{I}} \\
& f_{\mathrm{I}}^{\mathrm{DMD}}=\frac{\arg \left(\lambda_{\mathrm{I}}^{\mathrm{DMD}}\right)}{2 \pi \cdot \Delta t} \\
& g_{\mathrm{I}}^{\mathrm{DMD}}=\frac{\ln \left(\left|\lambda_{\mathrm{I}}^{\mathrm{DMD}}\right|\right)}{\Delta t}
\end{aligned}
$$

where $\mathbf{Y}$ and $\lambda^{\mathrm{DMD}}$ correspond to the eigenvectors and eigenvalues of $\mathbf{S}$. Each DMD mode $\boldsymbol{\phi}^{\mathrm{DMD}}$ contains a single frequency component $f^{\text {DMD }}$ associated to a particular growth rate $g^{\text {DMD }}$. Besides, a consequence of processing real-valued data is that the eigenvalues and associated eigenvectors are real or complex conjugates.

The above mathematical procedure holds for a full-rank data matrix $\mathcal{V}_{1}^{\mathrm{N}-1}$. For rank-deficiencies in the data, Schmid (2010) recommends a more robust SVD-based routine.

The time dynamics of the snapshots can be reconstructed on the basis of the DMD eigenvalues and modes by means of Eq.(3) and (9). However, the time coefficients first need to be known at all the time instants $\mathrm{k}$ or at the minimum at the first instant $\mathrm{k}=1$. Indeed, by identifying Eq.(3) and (9), it can be inferred that:

$$
\theta_{\mathrm{I}}^{\mathrm{DMD}}(\mathrm{k}+1)=\lambda_{\mathrm{I}}^{\mathrm{k}} \theta_{\mathrm{I}}^{\mathrm{DMD}}(1) \text { for } \mathrm{k}=1, \ldots, \mathrm{N}-1
$$

Since the DMD modes are not orthonormal, Tissot et al. (2013) have proposed projection methods to determine $\theta_{\mathrm{I}}(\mathrm{k})$. In the present work, the chosen technique is the one performing an orthogonal projection and using the Gram matrix of the modes $G=\phi^{\text {DMD }^{*}} \phi^{\text {DMD }}$. Making the inner product of Eq.(3) with the modes, it comes a linear system of equations whose matrix form can be written as:

$$
G \theta^{\mathrm{DMD}}=\phi^{\mathrm{DMD}^{*}} \mathcal{V}_{1}^{\mathrm{N}} \Rightarrow \theta^{\mathrm{DMD}}=G^{-1} \phi^{\mathrm{DMD}^{*}} \mathcal{V}_{1}^{\mathrm{N}}
$$

In other words, when snapshots stem from a non-linear process, as is the case for the present experiments, the dynamic modes represent the invariant structures of the best linear map from one snapshot to the next. When compared to POD, POD modes generally correspond to a mix of frequencies and are classified in terms of the amount of energy, and not as a function of their dynamical importance. This point is addressed by DMD analysis as DMD modes can isolate specific dynamic structures associated with a particular frequency. On the other hand, unlike POD, there is no single correct way to rank the eigenvalue importance. The determination of the most physically relevant DMD modes can be difficult without a knowledge of the underlying dynamics.

\section{Results and Discussion}

\subsection{Description of the flow field}

Following the Reynolds decomposition, the 2D-PIV instantaneous radial and axial velocity fields, $U_{r}(r, z, t)$ and $U_{z}(r, z, t)$ 
can be decomposed into a time-averaged mean component $\bar{U}(r, z)$ and a fluctuating component $u^{\prime}(r, z, t)$ :

$$
\begin{aligned}
& U_{r}=\overline{U_{r}}+u_{r}^{\prime} \\
& U_{z}=\overline{U_{z}}+u_{z}^{\prime}
\end{aligned}
$$

As the mean of the fluctuating components is by definition zero, it is normal practice to characterize them via their root mean square, $\sqrt{\overline{u_{r}^{\prime 2}}}(r, z)$ and $\sqrt{\overline{u_{z}^{\prime 2}}}(r, z)$.

The velocity fields are ensemble-averaged over 2175 (no binning -3 runs of 725) and 2900 (binning $2 \times 2-1$ run) instantaneous measurements to access the radial and axial components of the mean velocity and of the r.m.s. velocity. Actually, in a stirred vessel, the total r.m.s. velocity variations near the impeller include two contributions: a periodic component due to the impeller blade passage, and the true turbulent fluctuations of the overall flow field.

Fig.2(a) presents the magnitude of the velocity vectors $\sqrt{{\overline{U_{r}}}^{2}+{\overline{U_{z}}}^{2}}$ from an acquisition with no binning for $N=300 \mathrm{rpm}$, normalized by $N D$. These velocity fields reveal the typical flow pattern observed for a dual impeller Rushton turbine configuration: a stream is radially discharged from each turbine with a maximum velocity magnitude of $c a$. 1.8.ND. These flow streams are then swept up and down thanks to the baffles to form two large circulation loops in the vessel. The maps of the other acquisitions performed with another degree of resolution (binning $2 \times 2)$ and/or at different rotating speed $(N=150 \mathrm{rpm})$ are not reported for the sake of brevity since they display, as one might expect, similar mean velocity fields and circulation loops generated by the two impellers.

The dimensionless magnitude of the velocity vectors is shown for the vertical profile $r=0.55 D=0.055 \mathrm{~m}$ extracted from PIV data without binning and with a binning $2 \times 2$ at $N=300$ rpm in Fig.2(b) to study the effect of the change in spatial and time resolutions, and from PIV data acquired at $N=150$ and $300 \mathrm{rpm}$ without binning in Fig.2(c) to observe the impact of the rotating speed. The results show a very good agreement for both rotating speeds; all profiles are nearly overlaid on each other. The change of acquisition parameter values (spatial and time resolutions) has thus no impact on mean field calculations. Besides, as repeatedly pointed out in the literature, mean velocity profiles scale with impeller tip speed when operating in turbulent regime.

Fig.3 presents the radial $\sqrt{\overline{u_{r}^{\prime 2}}}$ and axial $\sqrt{\sqrt{u_{z}^{\prime 2}}}$ total r.m.s. velocity components from an acquisition at $N=300 \mathrm{rpm}$ with no binning $(\mathrm{a}, \mathrm{d})$. The fields shown are normalized by $N D$. The regions of highest r.m.s. fluctuating velocity fields for the axial and radial terms are in the impeller discharge. It is worth noting that PIV results may undergo some local inaccuracies because of the laser reflection on the free-surface and on the baffle located just behind the measurement plane. The two r.m.s components differ in certain zones, indicating that the turbulence is locally not isotropic. As for the mean velocity fields, the figures relevant to other acquisitions performed with another degree of resolution (binning $2 \times 2$ ) and/or at different rotating speed $(N$ $=150 \mathrm{rpm})$ are not displayed for the purpose of concision as they exhibit qualitatively similar patterns.
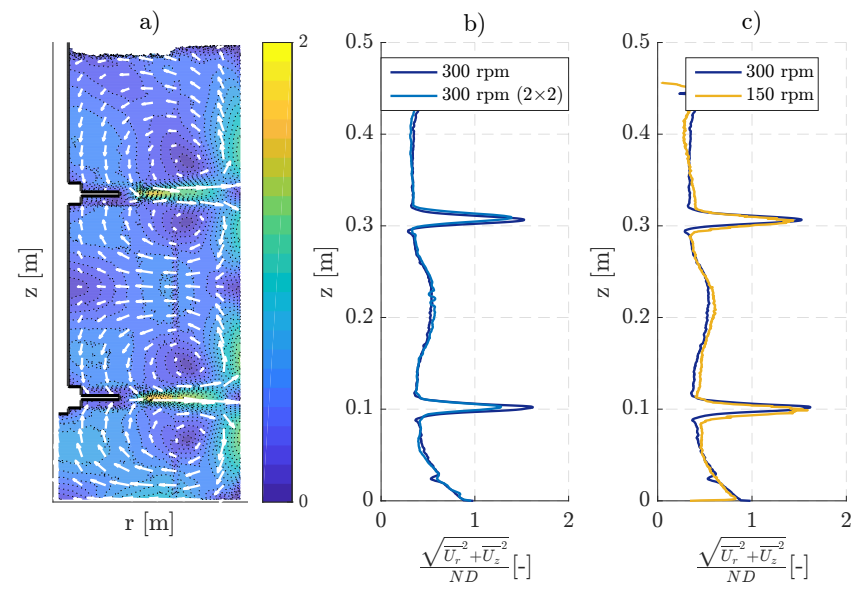

Figure 2: The dimensionless magnitude of the mean velocity vectors $\sqrt{{\overline{U_{r}}}^{2}+{\overline{U_{z}}}^{2}}$ on the measurement plane for $N=300 \mathrm{rpm}$ without binning, and its comparison on the vertical profile $r=0.55 D=0.055 \mathrm{~m}$ : for $N=300 \mathrm{rpm}$ without binning and with binning $2 \times 2$ (b), and for $N=150$ and $300 \mathrm{rpm}$ (c)

In Fig.3, the dimensionless radial (b, e) and axial (d, f) r.m.s. fluctuating velocity vectors are presented for the vertical profile $r=0.55 D=0.055 \mathrm{~m}$ extracted from PIV data. Data without binning and with a binning $2 \times 2$ at $N=300 \mathrm{rpm}$ are presented in Fig.3(b, c) to evaluate the effect of the change in spatial and time resolutions. The change of acquisition parameter values (spatial and time resolutions) does not significantly affect the accuracy of the kinetic energy measurement. Comparing PIV data acquired at $N=150$ and $300 \mathrm{rpm}$ without binning in Fig.3(e, f) allows for the impact of the rotating speed to be observed. There are discrepancies in the impellers jet. The r.m.s. fluctuations of each velocity component normalized by the blade tip velocity seems to decrease with increasing Reynolds number (Raju et al., 2005).

Fully-developed turbulence occurs when the inertial forces in the system are so large that the viscous forces become negligible. The accurate definition of this limit is critically dependent on appropriate choice of the characteristic length and velocity scales and is usually defined using the Reynolds number $R e$. The onset of fully-turbulent flow can be defined by means of the power number (constant) or the dimensionless velocity (mean or rms fluctuating component) profiles (collapse to a single similarity profile). The $R e$-independence characteristic of fully-developed turbulence can be considered as not completely fulfilled for $R e=2.4 \cdot 10^{4}$, which is also suggested by the slight difference in power number $\left(P_{0} 150 \mathrm{rpm}=5.6<5.9=P_{0} 300 \mathrm{rpm}\right)$. These observations can be related to some investigations (e.g., Raju et al. (2005), Machado et al. (2013)) that have been focused on Reynolds-number scaling of flow in stirred tanks and especially on criteria to determine the flow regime. The common threshold of $R e \geq 20000$ has to be considered with caution as, for instance, it may be valid only for regions close to the impeller. Besides, evaluating full turbulence based on either constant power number or kinematic similarity does not provide information about homogeneity of the turbulence in the tank, shape of profiles or size of fluctuating velocities.

Moreover, due to periodic disturbance of the vortices generated 
by the impeller blades, the measured fluctuation velocities near the impeller tip contained a large portion of periodic components. Wu and Patterson (1989) had shown that the periodic variations dominate the turbulence field close to the impeller, but rapidly diminish away from the tip. At $r=0.75 D$, it was reduced to about $20 \%$ of the total fluctuation. This was also the location where the random turbulence became fully-developed and had the highest intensity in the impeller stream. The conclusion from the present measurements is that the two rotating speeds do not correspond to the same flow regime. The difference in profiles of Fig. 3 may be explained partly by the dominance of periodic component for $N=150 \mathrm{rpm}$ and the increase in the truly turbulent fluctuations for $N=300 \mathrm{rpm}$. On top of that, although the finest spatial resolution (no binning) is adequate to estimate the Taylor micro-scale for both rotating speeds as detailed in Sect.2.2, it is clear that the smallest scales of turbulence $(\Delta x \approx \bar{\ell} \gg \bar{\eta})$ are more filtered at $N=300 \mathrm{rpm}$ than at $N=150 \mathrm{rpm}$. Similarly, as at $\mathrm{N}=300 \mathrm{rpm}$ the sampling frequency $f_{\text {acq }}=16 \mathrm{~Hz}$ is lower than the frequency of the vortex shedding $f_{\mathrm{BPF}}=20 \mathrm{~Hz}$, one can expect that all time variations have not been properly captured and consequently they have not been included in the evaluation of the r.m.s. velocity components. In other words, it is likely that turbulent fluctuations at $N=300 \mathrm{rpm}$ have not been fully captured compared to measurements performed at $N=150 \mathrm{rpm}$ because of the spatial and time resolutions of the present PIV system.

\subsection{Analysis of the flow field}

The two modal decomposition methods are applied to a single set of 725 (no binning) or 2900 (binning $2 \times 2$ ) liquid instantaneous radial and axial velocity fields together, without subtracting the mean flow from the data. The flow field inside the stirred tank is highly unsteady due to impeller rotation, including the periodic passage of the impeller blades in front of the baffles, and to turbulence. This section aims at giving an overview of the main characteristics of this flow field on the basis of the properties of their POD and DMD analyses. For this purpose, the investigation is presented in three parts: an overview of modal analysis using POD is given in Sect. 3.2.1 based on instantaneous velocity fields measured at $N=300$ rpm without binning, while the DMD technique is presented with the same dataset in Sect. 3.2.2. The influence of acquisition parameters (binning) and operating conditions (rotating speed) is then discussed in Sect.3.2.3.

Due to current limitations of PIV system in terms of time resolution, measurements are necessarily limited to the details they could capture. In stirred vessel, in the impeller region, the velocity field is characterized by two kinds of fluctuations: a periodic component due to the impeller blade passage, and the real turbulent fluctuations of the overall flow field. For $N=300$ $\mathrm{rpm}$, the organized periodic flow motion is consequently associated to the blade passage frequency $f_{\mathrm{BPF}}=\mathrm{N}_{\text {blades }} \cdot N=4 \cdot 5=20$ $\mathrm{Hz}$. Therefore, in the frame of signal analysis, data acquisition is not properly sampled. Indeed, the Nyquist sampling criterion requires that the data should be sampled at a rate that is at least twice the highest frequency to avoid aliasing effects (actual
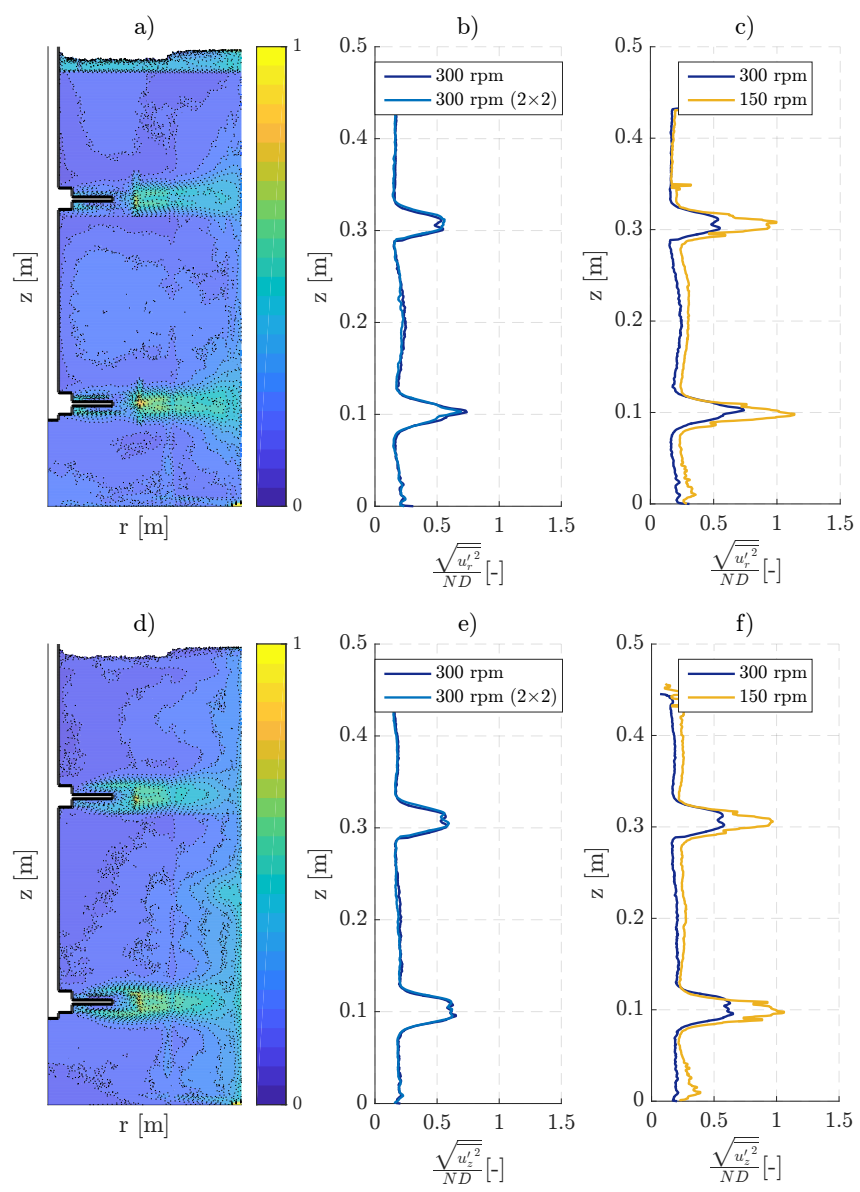

Figure 3: The dimensionless total r.m.s. velocity components $\sqrt{\overline{u_{r}^{\prime 2}}}$ (a) and $\sqrt{\overline{u_{z}^{\prime 2}}}$ (d) on the measurement plane for $N=300 \mathrm{rpm}$ without binning, and their comparisons on the vertical profile $r=0.55 D=0.055 \mathrm{~m}$ : for $N=300 \mathrm{rpm}$ without binning and with binning $2 \times 2(\mathrm{~b}, \mathrm{e})$, and for $N=150$ and $300 \mathrm{rpm}(\mathrm{c}, \mathrm{f})$ 
modification of frequencies). However, it should not be interpreted too pessimistically. In some cases, a signal can be sampled more slowly than the Nyquist frequency, especially when the nature of the signal is known. Besides, it is worth underlining that a steady repetitive signal has its energy concentrated in spikes around the fundamental frequency and its harmonics. The potential alias frequency $f_{\text {alias }}$ of the periodic signal related to the blade passage at the the sampling rate $f_{\text {acq }}=16 \mathrm{~Hz}$ is predicted by:

$$
f_{\text {alias }}=\left|f_{\mathrm{BPF}}-\left(n \cdot f_{\mathrm{acq}}\right)\right|
$$

where $\left(n \cdot f_{\text {acq }}\right)$ is the integer multiple of the sample rate closest to the input signal frequency. The $20 \mathrm{~Hz}$ signal, sampled at 16 $\mathrm{Hz}$, is expected to be masquerading as a $4 \mathrm{~Hz}$ signal.

In other words, considering harmonic interference in general, the acquisition and the motion of the 4-blades Rushton turbines can be basically seen as two harmonic signals with slightly different frequencies that are superimposed. The interference between the two signals, known as the beats, alternate between constructive and destructive. In any case, this interference term oscillates with beat frequency $f_{\text {beat }}=|\Delta f|=\left|f_{\text {BPF }}-f_{\text {acq }}\right|$.

\subsubsection{Proper orthogonal decomposition}

The POD eigenvalues correspond to the contribution of each mode to the total kinetic energy in the measurement plane (2D domain). Fig.4 shows the energy content of the modes, expressed as a percentage of the total energy contained in all modes together from data acquired at $N=300 \mathrm{rpm}$ without binning.

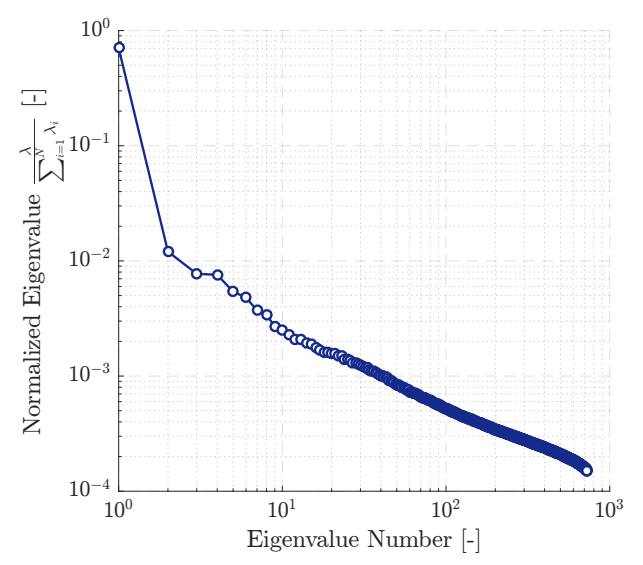

Figure 4: POD eigenvalues spectrum of velocity fields for $N=300 \mathrm{rpm}$ without binning $(\Delta t=0.0625 \mathrm{~s}(16 \mathrm{~Hz}), \mathrm{N}=725)$

The time behavior of a mode is described by the POD time coefficients $\theta_{i}(t)$. Fig.5 shows the time coefficients of five POD modes $(1,2,3,5$ and 6$)$ for $N=300 \mathrm{rpm}$ without binning while Fig. 6 shows the frequency content of these time coefficients. The frequency analysis is performed using the Lomb-Scargle method as it is able to detect a periodic signal in noisy data and to accurately determine the single and multiple frequencies that may be expected or not (Roussinova et al., 2004).

Although these results have a quite large time step $(\Delta t=0.0625$ $\left.\mathrm{s}\left(f_{\mathrm{acq}}=16 \mathrm{~Hz}\right)\right)$, they deemed adequate to capture the dominant flow behavior. The interference pattern between acquisition and impeller blade motion accounts for the frequency content of PIV data, i.e. the presence of peaks at $f=4 \mathrm{~Hz}$ and its overtones. However, the periodic behavior of the flow generated by the impellers is more complex (not a single frequency) and small-scale structures are present within the tank. PIV experiments give more detail while identifying another frequency besides $f_{\mathrm{BPF}}$ as the time coefficients $\theta_{5}(t)$ and $\theta_{6}(t)$ have clear peaks at $f=5 \mathrm{~Hz}=N$.

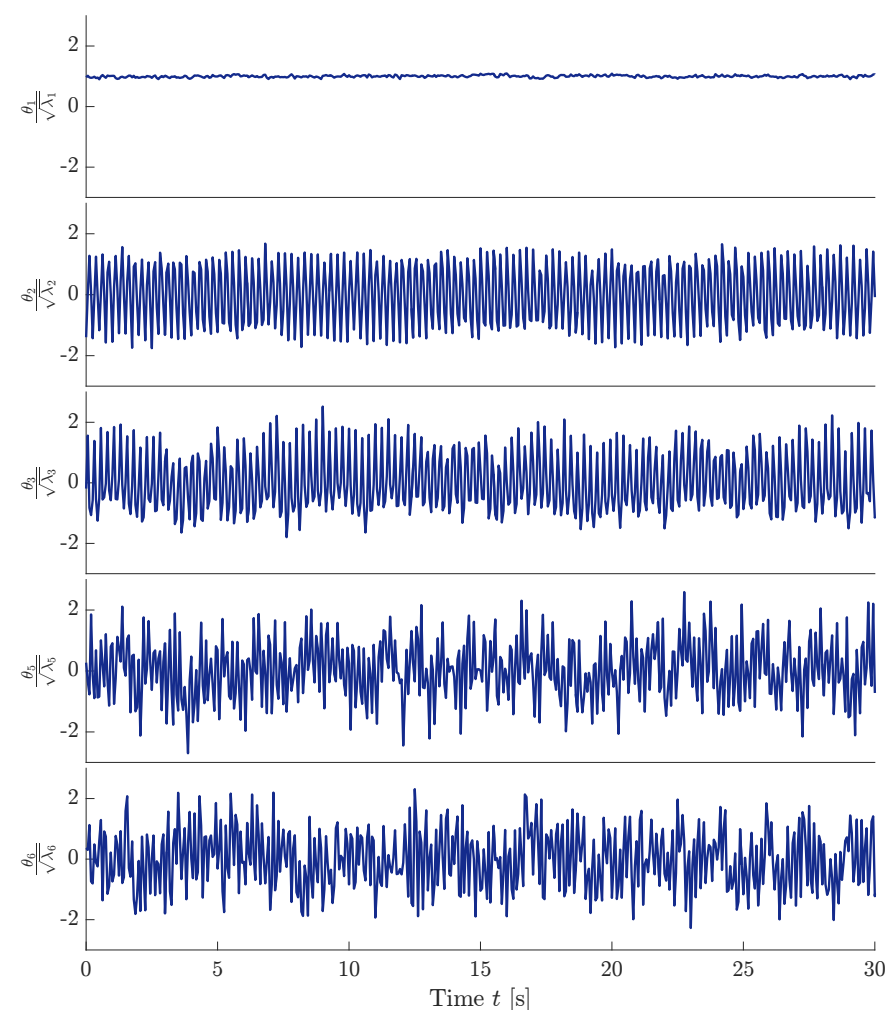

Figure 5: Comparison of the POD coefficients $\theta_{i}(t)$ based on their time evolution ( $\mathrm{i}=1,2,3,5$ and 6$)$ for $N=300 \mathrm{rpm}$ without binning $(\Delta t=0.0625 \mathrm{~s}(16$ $\mathrm{Hz}), \mathrm{N}=725$ )

The first POD mode (POD mode 1), which is characteristic of the mean flow contribution, contains approximately $70 \%$ of the total energy. The time coefficient corresponding to POD mode $1 \theta_{1}(t)$ is approximately constant as shown in Fig.5 and consequently the associated periodogram in Fig. 6 highlights no specific frequencies.

The velocity field associated to this first mode can be reconstructed and compared to the statistical average of the 2D-PIV instantaneous velocity fields (Eq.(21)). In Fig.7 are presented, for instance, the magnitude of the mean velocity vectors normalized by $N D$ obtained from PIV (a) and the velocity field reconstructed from POD mode 1 (b) for the set of 725 snapshots with a sampling frequency of $16 \mathrm{~Hz}$. The dimensionless magnitude of the velocity vectors is also reported for the vertical profile $r=0.055 \mathrm{~m}$ for PIV and POD data (c). The results are identical; the velocity field associated to the first POD mode fully corresponds to the mean flow. 


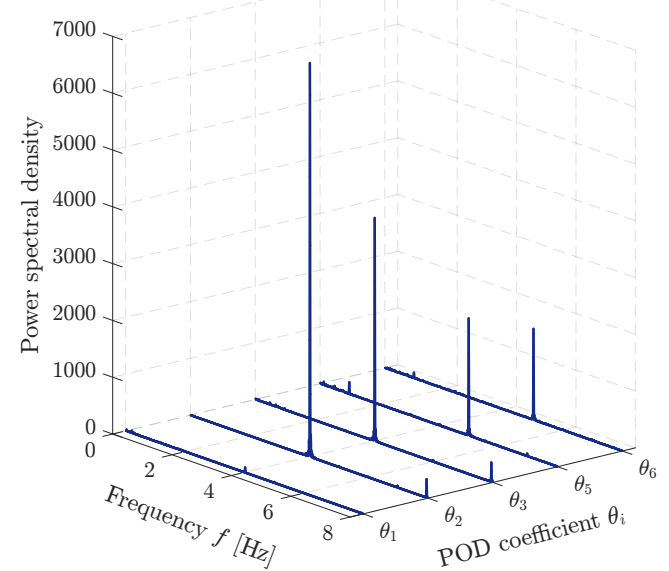

Figure 6: Comparison of the POD coefficients $\theta_{i}(t)$ based on their LombScargle periodogram ( $\mathrm{i}=1,2,3,5$ and 6$)$ for $N=300 \mathrm{rpm}$ without binning $(\Delta t=0.0625 \mathrm{~s}(16 \mathrm{~Hz}), \mathrm{N}=725)$ a)

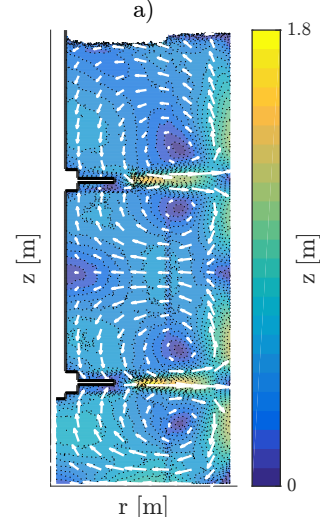

b)

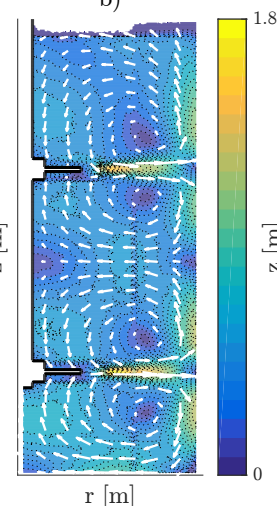

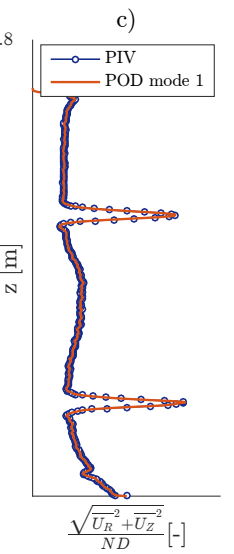

Figure 7: Comparison of the dimensionless magnitude of the mean velocity vectors on the measurement plane (a,b) and on the vertical profile $r=0.055 \mathrm{~m}$ (c) for $N=300 \mathrm{rpm}$ without binning $(\Delta t=0.0625 \mathrm{~s}(16 \mathrm{~Hz}), \mathrm{N}=725)$ : PIV $\sqrt{{\overline{U_{r}}}^{2}+{\overline{U_{z}}}^{2}} / N D$ (a), POD mode $1-\sqrt{\left(\overline{\theta_{1}} \phi_{r, 1}\right)^{2}+\left(\overline{\theta_{1}} \phi_{z, 1}\right)^{2}} / N D$ (b)

The remaining modes are related to the fluctuating part of the flow field (Reynolds decomposition: Eq.(21)). Previous studies (e.g. Ducci et al. (2008), Liné et al. (2013), Liné (2016)) have shown that POD makes it possible to decompose the fluctuating term into an organized periodic component associated to the impeller rotation and a random turbulent component (triple decomposition). Although a first inspection of the spectrum show no obvious coupling between some of the first modes, their time evolution contains more information and reveals interesting features.

The frequency analysis of the time coefficients of the first other POD modes in Fig.6 and their spatial distribution in Fig.8 reveal that the second and third modes (POD modes 2 and 3) come in pairs, as well as the fifth and sixth modes (POD modes 5 and 6).

Indeed, as each pair of these modes has eigenvalues with nearly the same norm, both components of a pair are thus equally important in terms of their contribution to the overall energetic

budget. This feature can be explained by the periodicity of the flow. Each pair of associated modes corresponds to similar but phase-shifted fluid structures (Ducci et al. (2008), Liné et al. (2013)). This is depicted in Fig.8.(a-b, c-d) where the magnitude of these POD modes $\left(\sqrt{\left(\phi_{r, i}\right)^{2}+\left(\phi_{z, i}\right)^{2}}\right)$ is shown. The structures of POD modes 2 (a) and 5 (c) appear displaced relative to the structures of POD modes 3 (b) and 6 (d) respectively. The POD modes 2 and 3 exhibit symmetrically-organized structures with respect to each Rushton turbine plane and correspond to the pair of trailing vortices induced by the impeller blades. Indeed, because of the presence of a disk, two vortices are generated behind a Rushton turbine: above and below the disk. POD modes 5 and 6 present vortical structures in the zone of the impeller discharge flow. These four modes correspond to organized motions generated by the impeller rotation. The analysis of the corresponding time coefficients confirms these similarities between each couple of structures (Fig.5): POD coefficients 2 and 5 show periodicity with peak amplitudes similar to those of POD coefficients 3 and 6 respectively. As above-mentioned, the frequency analysis of the signal from $\theta_{i}(t)$ (Fig.6) shows that there are very clear peaks at specific frequencies. $\theta_{2}(t)$ and $\theta_{3}(t)$ have frequencies of 4 and $8 \mathrm{~Hz}$ while $\theta_{5}(t)$ and $\theta_{6}(t)$ have a frequency of $5 \mathrm{~Hz}$. The occurrence of these frequencies can be explained by the impeller geometry and their rotation: 4 and 8 $\mathrm{Hz}$ are the blade passage frequency and its upper partial, and 5 $\mathrm{Hz}$ is the impeller rotating speed.
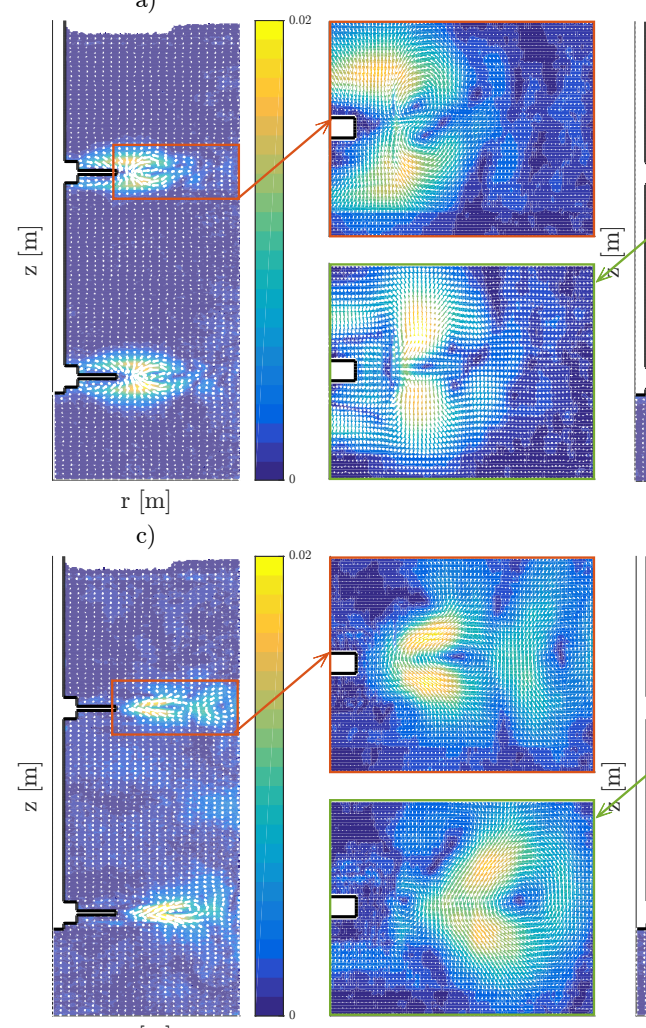

$\mathrm{r}[\mathrm{m}]$ b)

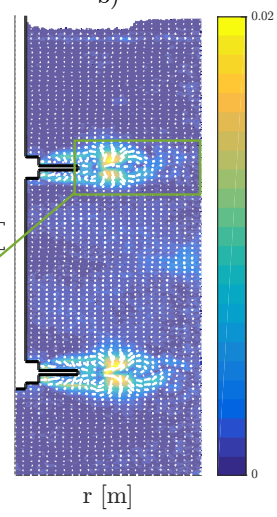

d)

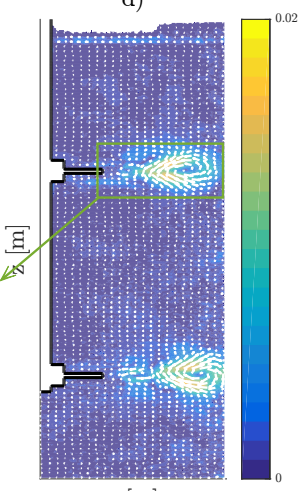

$\mathrm{r}[\mathrm{m}]$
Figure 8: Comparison of the magnitude of the POD modes on the measurement plane $\sqrt{\left(\phi_{r, i}\right)^{2}+\left(\phi_{z, i}\right)^{2}}(\mathrm{i}=2(\mathrm{a}), 3(\mathrm{~b}), 5$ (c) and $6(\mathrm{~d}))$ for $N=300 \mathrm{rpm}$ without binning $(\Delta t=0.0625 \mathrm{~s}(16 \mathrm{~Hz}), \mathrm{N}=725)$ 
The magnitude of POD mode 4 is also shown in Fig.9(a); its contour plot suggests that it corresponds to the mean interaction between the two Rushton turbines and the generated trailing vortices. Indeed, three zones of recirculation are identified: between the two impellers and one at each impeller tip. The analysis of the related time coefficient in spectral space (Fig.9(c)) presents oscillations near zero that may be seen as oscillations around the mean field but also reveals the presence of the frequencies of 4 and $5 \mathrm{~Hz}$. However, the peaks have quite lower magnitudes if compared to those observed for POD modes 2, 3, 5 and 6 . It is worth noting that, as a consequence of its optimization principle, POD does not necessarily separate the flow into its frequency content. Therefore, one mode often encompasses several frequencies and several physical phenomena.

To illustrate that the higher eigenvalues (POD modes $\mathrm{i}>6$ ) display no specific spatial structure and are thus related to turbulent flows, the contour plot of the magnitude of POD mode 50 is shown in Fig.9(d) It exhibits no particular coherent pattern and is rather random and chaotic. As shown in Fig.9(f), $\theta_{50}(t)$ displays no specific temporal behavior as expected for a turbulent flow.
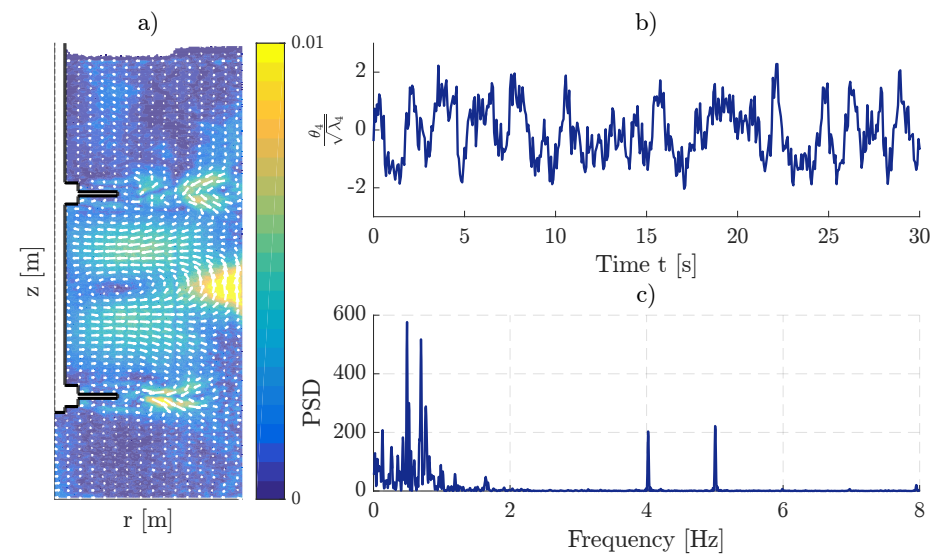

d)
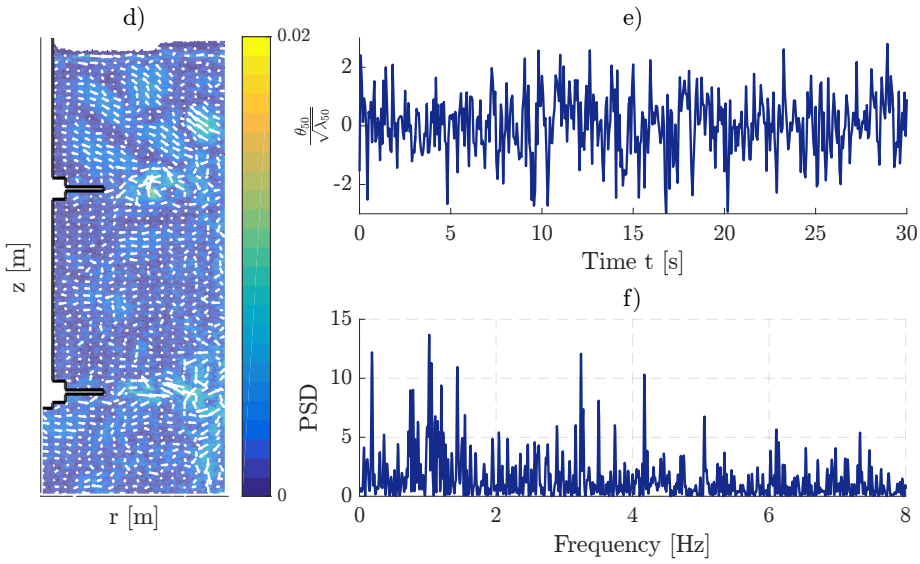

Figure 9: Magnitude of the POD modes 4 (a) and 50 (d) for $N=300$ rpm without binning $(\Delta t=0.0625 \mathrm{~s}(16 \mathrm{~Hz}), \mathrm{N}=725)$, along with their time coefficients (4 - b, 50 - e) and their frequency content (4 - c, 50 - f)

\subsubsection{Dynamic mode decomposition}

In principle, the DMD results allow evidencing the same phenomena as the POD analysis. As already mentioned, DMD enables the retrieval of spatial structures at given frequencies.

The aim of this section is therefore to provide a description of the most pertinent modes identified using the DMD analysis and to compare them with the modes singled out by the POD study.

Without any explicit determination of the underlying dynamical operator, the DMD algorithm determines eigenvalues and eigenvectors of an approximate linear model. Results of datasets at $N=300 \mathrm{rpm}$ without binning are shown in Fig.10.

The eigenvalues $\lambda_{\mathrm{I}}$ contain information about the dynamical behavior of their corresponding modes $\phi_{\mathrm{I}}$. To reveal these temporal data, the eigenvalues are transformed into frequencies $f_{\mathrm{I}}$ and associated growth/decay rate $g_{\mathrm{I}}$ using Equations 17 and 18 . DMD results in the discrete spectra $\left(\mathfrak{J}\left(\lambda_{\mathrm{I}}\right) v s . \mathfrak{R}\left(\lambda_{\mathrm{I}}\right)\right)$ are displayed in Fig.10(a), while those in the continuous spectra $\left(g_{\mathrm{I}}\right.$ vs. $f_{\mathrm{I}}$ ) are shown in Fig.10(b).

The Euclidian norm of the dynamic modes can be used to sort them along with their associated frequencies, which gives a basis for sorting them with respect to their relevance as the amplitude spectrum helps identify the modes associated with the most physically relevant phenomena. The relative importance of each spatial structure is described in the amplitude distribution $\left(\log \left\|\Phi_{\mathrm{I}}\right\| v s . f_{\mathrm{I}}\right)$ in Fig.10(c).

However, additional information has also to be extracted from the growth/decay rate of each mode as a mode with a high amplitude can be strongly damped. In order to assess the findings based on the Euclidian norm of the dynamic modes, an energetic criterion $E_{\mathrm{I}}$ introduced by Tissot et al. (2014) is used in which the amplitude of the mode is weighted by its time coefficient:

$$
E_{\mathrm{I}}=\frac{1}{T} \int_{0}^{T}\left\|\phi_{\mathrm{I}} \lambda_{\mathrm{I}}^{t / \Delta t}\right\| \mathrm{d} t=\frac{e^{2 g_{\mathrm{I}} T}-1}{2 g_{\mathrm{I}} T}
$$

where $T$ is the time-horizon of the snapshots, i.e. $T=(\mathrm{N}-1) \Delta t$. The spectra based on this energy criterion $\left(\log \sqrt{E}_{\mathrm{I}} v s . f_{\mathrm{I}}\right)$ are presented in Fig.10(d).

In the eigenvalue spectra in Fig.10(a), the size and the color (from yellow $=$ low to blue $=$ high) of the symbol (circle marker) associated to each eigenvalue indicate the amplitude of the respective structure in the dataset. The spectra are symmetric with respect to the imaginary axis as eigenvalues come in complex conjugated pairs. Nearly all eigenvalues lie close to the unit circle $|\lambda|=1$. The unitary circle itself represents stable flow structures, while points inside the unit circle represent dynamically decaying structures and points outside it represent dynamically growing structures. It is thus said that the data are lying near an attractor. It is worth noting that it could be expected that the modes of a fully developed turbulent flow should approach the unit circle (Semeraro et al., 2012). Indeed, when the limits of fully developed turbulence in the bulk of the tank are based on the the scaling approach (mean or r.m.s. velocity profiles), the comparison of the data require time-averaged (ensemble-averaged) values in the tank. From a practical standpoint, the mean and variance of the flow are 

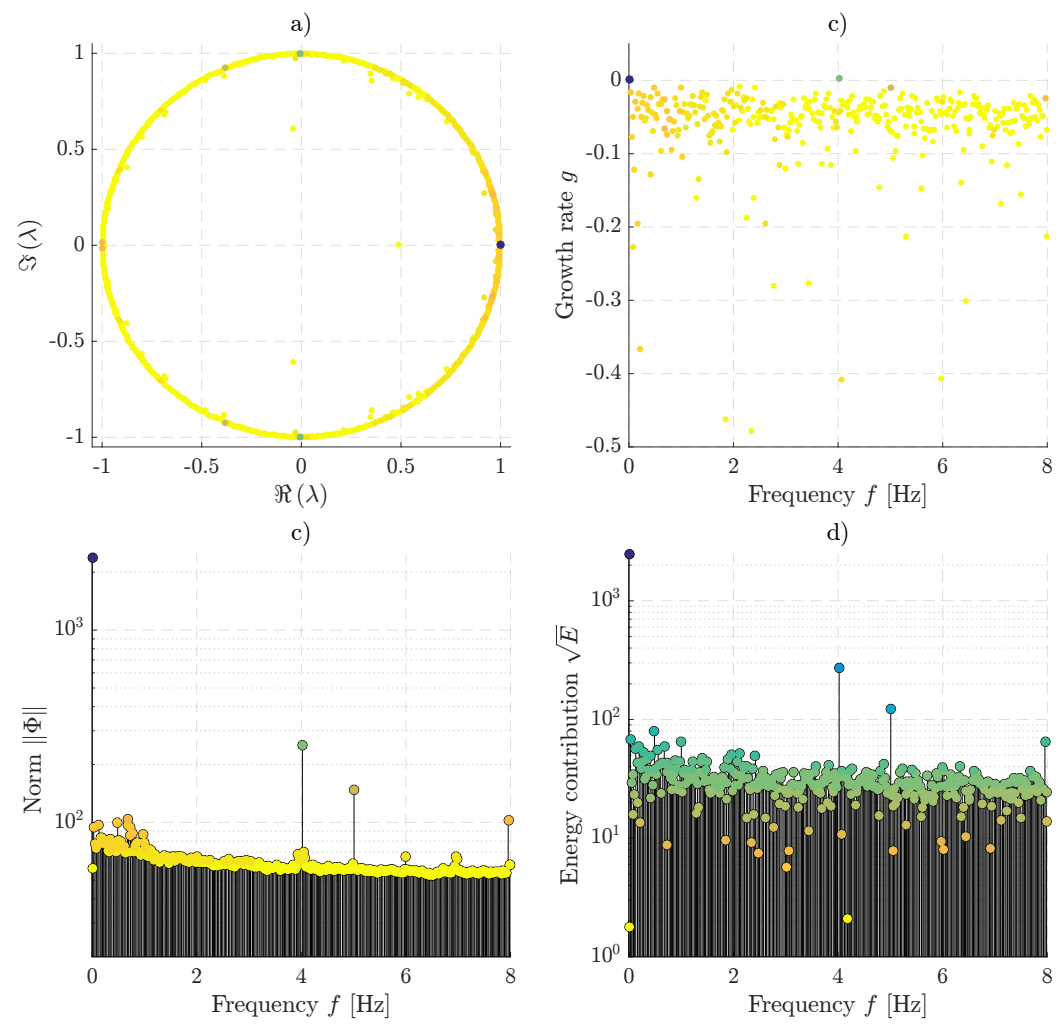

Figure 10: Dynamic mode spectra for $N=300 \mathrm{rpm}$ without binning $(\Delta t=0.0625 \mathrm{~s}(16 \mathrm{~Hz}), \mathrm{N}=725)$ : eigenvalues $\lambda$ spectra (a), growth rate $g$ s. frequency $f$ spectra (b), norm $\|\Phi\| v s$. frequency $f$ spectra (c), energy contribution $\sqrt{E} v s$. frequency $f$ spectra (d)

considered as statistically stationary. This does not mean that there are no random-fluctuations in velocity but simply that the time means and variances at any given point in the tank are independent of time, when estimated from a sufficient number of measurements, taken over a sufficient long interval of time.

In the $f-g$ spectra plotted in Fig.10(b), the symbol size and color of the data also report the amplitude of the associated mode. They show a strong alignment along the horizontal neutral line, i.e. the zero growth limit $(g=0)$ which represents the persistent or statistically stationary processes. Values in the negative half plane represent decaying structures while values in the positive one represents growing or expanding structures. On these spectra, one can observe an eigenvalue at the origin $(f$ $=0 \mathrm{~Hz}$ ), which reveals the existence of a corresponding structure that is steady in time. It is the only one which is represented by a real function. The associated dynamic mode corresponds to the structure of the mean flow. All the other modes are complex, indicating that they are travelling wave-like structures.

The Euclidean norms $\|\Phi\|$ are shown in Fig.10(c) with the same coloring as for the spectra in Fig.10(a) and (b). Each mode is displayed with a vertical line scaled with the logarithm of its amplitude at its corresponding frequency. Ordering the modes with respect to the norms, the first three strongest unsteady modes after the mean flow oscillate at $f=4,5$ and $8 \mathrm{~Hz}$, respectively. This is in agreement with the POD results.

In general, the amplitudes are not necessarily a criterion of dynamic relevance. As above-mentioned, modes with very high amplitudes can be very quickly damped. However, in the present study, the most amplified modes correspond to the most persistent ones. Fig.10(d) represents the energy contribution of each DMD mode. Modes associated to frequencies other than $f=4,5$ and $8 \mathrm{~Hz}$, are thus likely related to significantly less relevant dynamic structures.

Fig.11 shows the comparison between the time coefficients of the DMD modes $\theta$ associated to $f=0,4,5$ and $8 \mathrm{~Hz}$ for $N=$ $300 \mathrm{rpm}$ without binning.

The dynamic mode associated to $f=0 \mathrm{~Hz}$ represents the structure of the mean flow. The corresponding time coefficient $\theta$ $0 \mathrm{~Hz}$ is thus approximately constant as shown in Fig. 11 for $N$ $=300 \mathrm{rpm}$ without binning. As for POD, the velocity field associated to this first mode can be reconstructed and compared to the statistical average of the 2D-PIV instantaneous velocity fields. As an example, Fig.12 presents the magnitude of the velocity vectors normalized by ND from PIV (a) and DMD (b) for $N=300 \mathrm{rpm}$ without binning. The dimensionless magnitude of the velocity vectors is also reported for the vertical profile $r=0.055 \mathrm{~m}$ for DMD and PIV data (c). The two profiles are superimposed which confirms that the velocity field associated to the first DMD mode exactly corresponds to the mean flow.

The DMD modes - $f=4,5$ and $8 \mathrm{~Hz}$ represent the main dynamic structures and thus contain interesting information on flow features. As illustrated by their time coefficients in Fig.11, the imaginary parts of these three modes are phase-shifted compared to the associated real ones. Furthermore, the periodic behavior of the DMD modes - $f=4$ and $5 \mathrm{~Hz}$ is well-captured and the DMD mode $-f=8 \mathrm{~Hz}$ is clearly damped. Moreover, unlike 


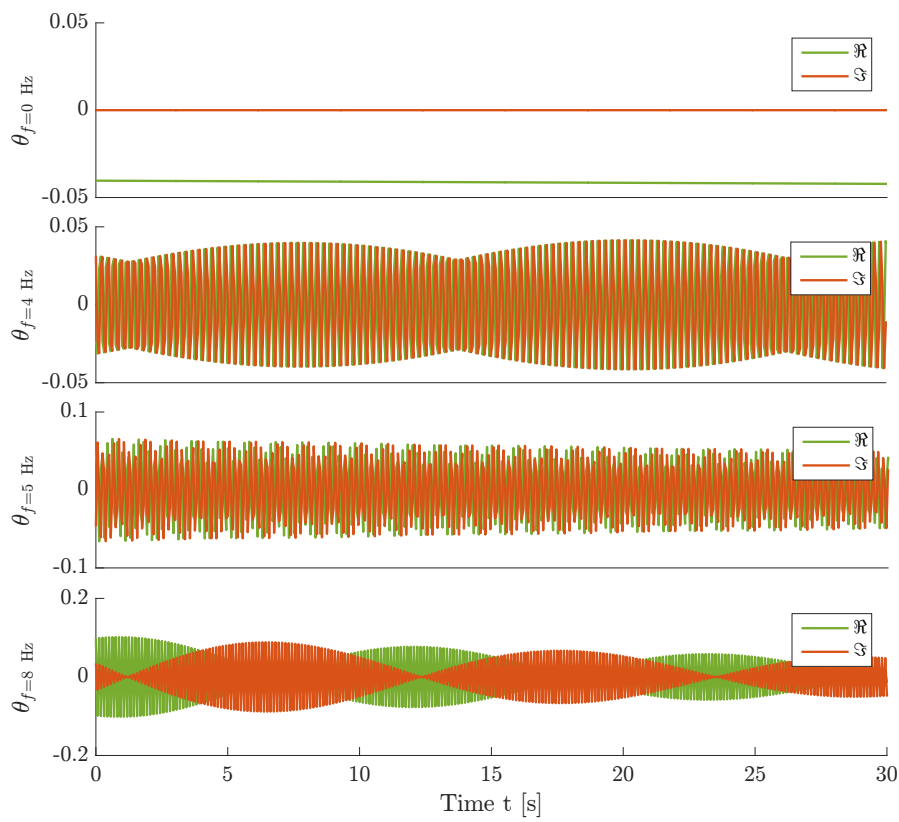

Figure 11: Comparison of the time coefficients of the DMD modes $\theta$ associated to $f=0,4,5$ and $8 \mathrm{~Hz}$ for $N=300 \mathrm{rpm}$ without binning ( $\Delta t=0.0625 \mathrm{~s}(16$ $\mathrm{Hz}), \mathrm{N}=725$ )

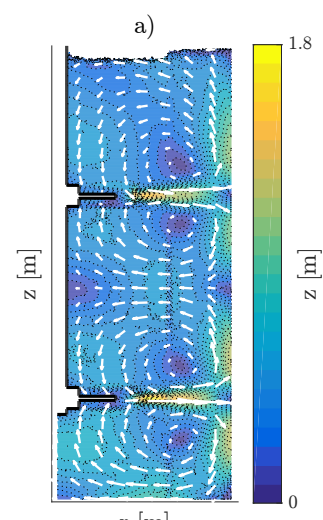

$\mathrm{r}[\mathrm{m}$ b)

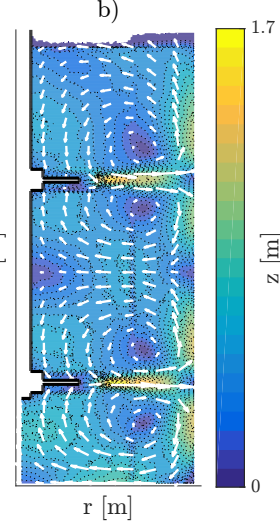

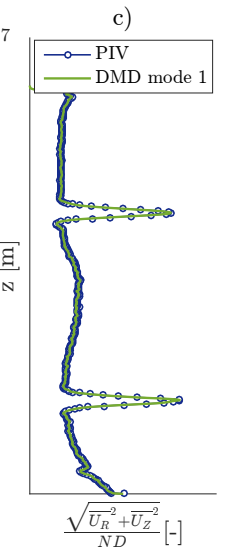

Figure 12: Comparison of the dimensionless magnitude of the mean velocity vectors on the measurement plane (a,b) and on the radial profile $r=0.055 \mathrm{~m}$ (c) for $N=300 \mathrm{rpm}$ without binning $(\Delta t=0.0625 \mathrm{~s}(16 \mathrm{~Hz}), \mathrm{N}=725)$ : PIV $\sqrt{{\overline{U_{r}}}^{2}+{\overline{U_{z}}}^{2}} / N D$ (a) and DMD mode $-f=0 \mathrm{~Hz}-\sqrt{\left(\overline{\theta_{1}} \phi_{r, 1}\right)^{2}+\left(\overline{\theta_{1}} \phi_{z, 1}\right)^{2}} / N D$ (b)

$\theta_{f=5 \mathrm{~Hz}}$, the coefficients $\theta_{f=4 \mathrm{~Hz}}$ and $\theta_{f=8 \mathrm{~Hz}}$ exhibit an interference pattern with a time period of about $12 \mathrm{~s}$. Consequently, contrary to POD, the DMD method differentiates the real frequencies from the alias ones. The amplitude variations of the DMD time coefficients associated with $f_{\mathrm{BPF}}$ is the consequence of the modal decomposition attempt to replicate the original signal. Indeed, considering a signal oscillating at exactly $20 \mathrm{~Hz}$ and sampled at exactly $16 \mathrm{~Hz}$, a same point is sampled every five cycles. This said, if the signal is sampled at a frequency slightly different from $16 \mathrm{~Hz}$, the signal phase is advancing a little bit with each new sampling. With time, the effect is then a replica of of the input signal, only slower.

Fig.13 shows the magnitude of real (a, c, e) and imaginary (b, $\mathrm{d}, \mathrm{f})$ parts of the dynamic modes $\sqrt{\left(\phi_{r, i}\right)^{2}+\left(\phi_{z, i}\right)^{2}}$ corresponding to these three dominant frequencies $(f=4,5$ and $8 \mathrm{~Hz})$. The field based on the real part of the DMD mode - $f=4 \mathrm{~Hz}$ (Fig.13(a)) shows symmetrically organized structures with respect to each Rushton turbine plane, which correspond to the pattern of the trailing vortices also detected for POD modes 2 and 3. The field based on the real part of the DMD mode $-f=5 \mathrm{~Hz}=$ $N$ (Fig.13(c)) displays vortical structures at each impeller discharge flow, which is a pattern quite similar to the ones detected for POD modes 5 and 6 . The field based on the real part of dynamic mode - $f=8 \mathrm{~Hz}$ (Fig.13(e)) has smaller coherent features concentrated in the immediate surroundings of each impeller than the one associated to $f=4 \mathrm{~Hz}$ (reminiscent of higher harmonic). These modes closely resemble the POD modes shown in Fig.8, which are characterized by well-defined frequencies that are matching the ones identified by the DMD.

These results show that DMD is a powerful tool for isolating structures when distinct frequencies are present in the flow field. With this method, it is possible to extract information on the flow that is otherwise hard to visualize due to the interaction and to the superposition of different phenomena characterized by different dynamic behavior.

One has to keep in mind that the structures evidenced by POD analysis are those characterized by a given energy level, while structures associated to DMD modes are characterized by a given frequency. In general, DMD and POD analyses should thus lead to different, but complementary, information about the flow. These results agree with the view suggested by Schmid et al. (2012) and Semeraro et al. (2012): the modes obtained with the two methodologies tend to be similar when the flow behaves as a periodic oscillator, which is the case in the present study. For instance, on the one hand, the DMD technique is superior to POD if one is interested in the flow behavior at certain frequencies (extraction of the spatial structures associated to these dominating frequencies), e.g. time variations of the mean flow (macro-instabilities) and other instability mechanisms. On the other hand, POD method is more appropriate than DMD if the study is related to the energy transfer between the different characteristic length scales (from the large to the turbulent eddies), e.g. the rate of total viscous dissipation of kinetic energy and the shear rate.

\subsubsection{Influence of acquisition parameters and operating con- ditions}

In Sect.3.2.1 and 3.2.2, it has been shown that POD and DMD are powerful analysis tools to evidence spatio-temporal structures from instantaneous flow fields measured by PIV. In Sect.3.2.3, the aim is to study the potential influence of PIV acquisition parameters (binning) and operating conditions $(N)$ on the results of those analyses.

The POD spectra in Fig.14 show the normalized energy for different acquisition parameters (a: $N=300 \mathrm{rpm}$ acquired without and with binning $2 \times 2$ ), and operating conditions (b: $N=$ $300 \mathrm{rpm}$ and $150 \mathrm{rpm}$ with binning $2 \times 2$ ).

Both sets of snapshots acquired at $N=300 \mathrm{rpm}$ (Fig.14(a)) are adequate to resolve the structures of the present flow case as they are in perfect agreement for the first two decades of modes. 
a)

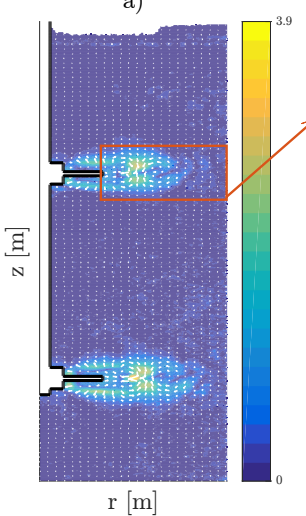

c)

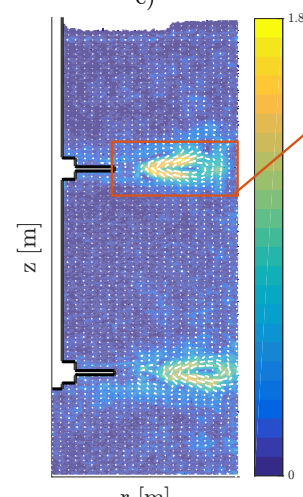

$\mathrm{r}[\mathrm{m}]$

e)

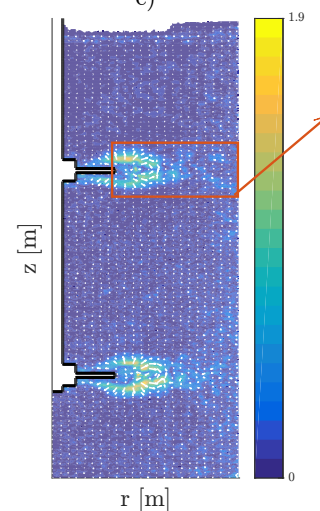

$\mathrm{r}[\mathrm{m}]$
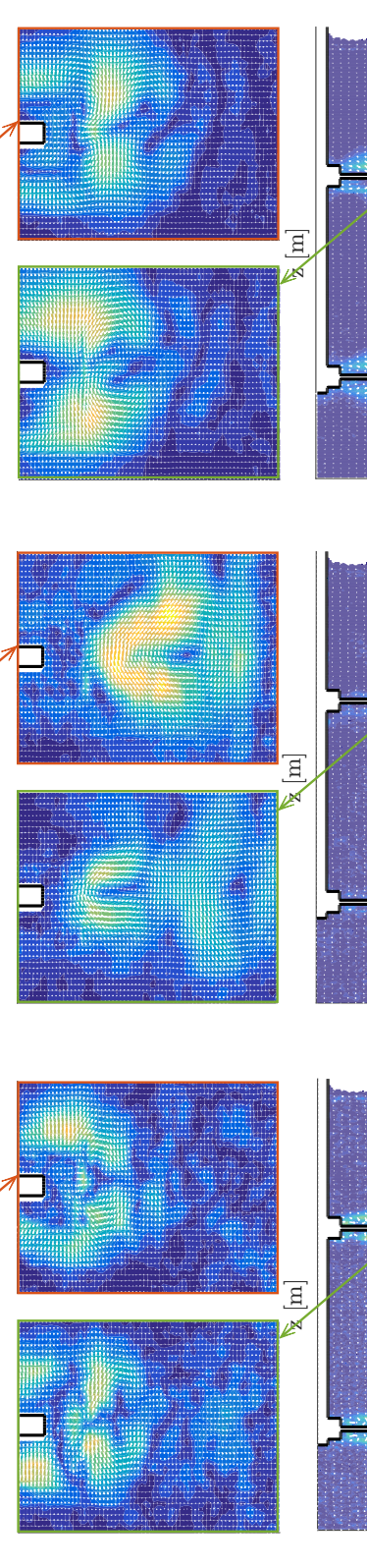

b)

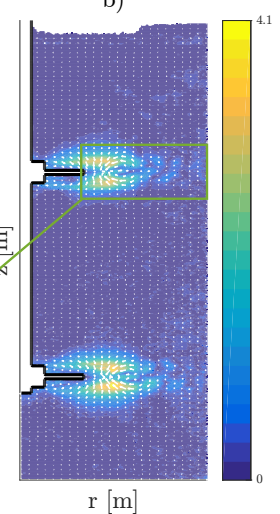

d)

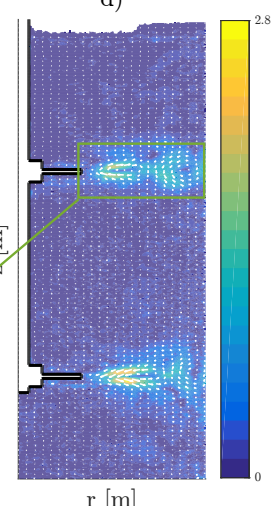

f)

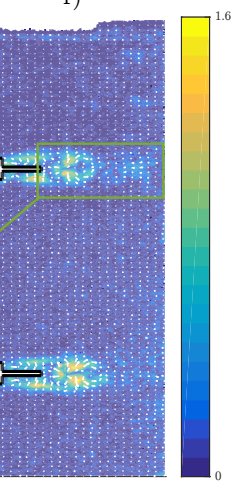

$\mathrm{r}[\mathrm{m}]$
Figure 13: Comparison of the magnitude of the DMD modes associated to $f$ $=4(\mathfrak{R}(\mathrm{a})$ and $\mathfrak{I}(\mathrm{b})), 5(\mathfrak{R}$ (c) and $\mathfrak{J}(\mathrm{d}))$ and $8 \mathrm{~Hz}(\mathfrak{R}(\mathrm{e})$ and $\mathfrak{J}(\mathrm{f}))$ on the measurement plane $\sqrt{\left(\phi_{r, i}\right)^{2}+\left(\phi_{z, i}\right)^{2}}$ for $N=300 \mathrm{rpm}$ without binning $(\Delta t=0.0625$ $\mathrm{s}(16 \mathrm{~Hz}), \mathrm{N}=725)$

They are deviating for the upper modes which contribute to lower levels of total kinetic energy and depend on acquisition parameters, especially on the number of snapshots $\mathrm{N}$ (no binning: 725 images $v s$. binning $2 \times 2$ : 2900 images) in the present case.

Whatever the rotating speed $N=150$ or $300 \mathrm{rpm}$, the variation of the kinetic energy associated with the modes (Fig.14(b)) evolves similarly. The first mode, corresponding to the mean flow field, explains the major percentage of the total kinetic energy; each of the following modes contains less kinetic energy. Moreover, it can be seen that the upper modes for $N=300$ rpm follow a linear trend (black dashed line) in the log-log plot

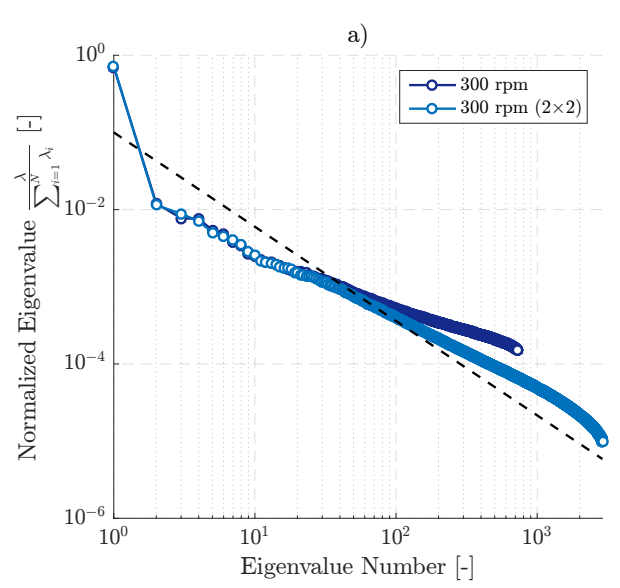

b)

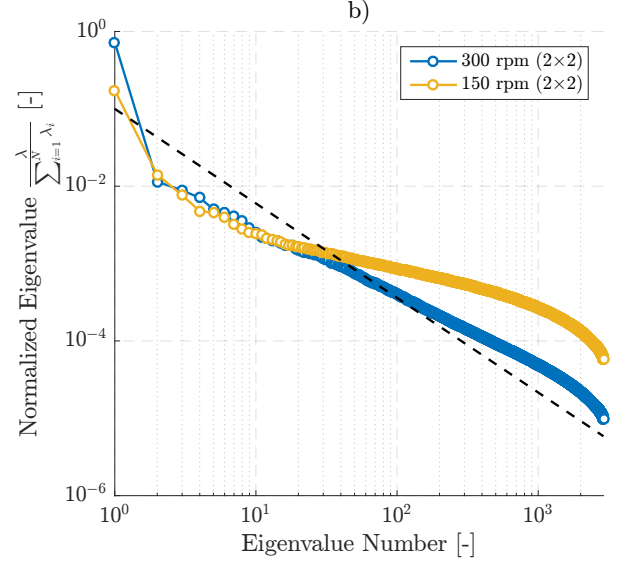

Figure 14: POD eigenvalues spectra of velocity fields for $N=300 \mathrm{rpm}$ without binning and with binning $2 \times 2$ (a), and for $N=300$ and $150 \mathrm{rpm}$ with binning $2 \times 2(b)$

(-11/9), contrary to the lower rotating speed. A (-11/9) power scaling is a characteristic of inertial range of turbulence (Liné et al. (2013), Liné (2016)). The discrepancy can be explained by the limitation of $2 \mathrm{D}$ measurement of a $3 \mathrm{D}$ velocity field and the filtering of the smallest scales of turbulence as $\Delta x \approx \bar{\ell} \gg \bar{\eta}$.

The organized flow motion is associated with the blade passage frequency $f_{\mathrm{BPF}}=10$ and $20 \mathrm{~Hz}$ for $N=150$ and $300 \mathrm{rpm}$ respectively. According to the Nyquist sampling criterion, PIV data acquired with a binning $2 \times 2$ at $f_{a c q}=24 \mathrm{~Hz}$ are properly sampled for $N=150 \mathrm{rpm}$ compared to the impeller blade motion, and are again associated with an alias frequency of $4 \mathrm{~Hz}$ for $N=300 \mathrm{rpm}$ (Eq.(22)). The trailing vortices generated by the Rushton blades are then expected at $f=10$ and $4 \mathrm{~Hz}$ for $N=150$ and $300 \mathrm{rpm}$ respectively, for PIV measurement with binning. It is worth noting that the Nyquist criterion does not provide the optimal sampling time interval but the minimum one to avoid aliasing effects.

Fig. 15 shows the frequency content of the first six time coefficients and of the fiftieth one, which are the same POD modes as analyzed in Sect.3.2.1.

In Fig.15(a), the POD data obtained at $N=300 \mathrm{rpm}$ for both PIV acquisition parameters $(16 \mathrm{~Hz}$ without binning, and $24 \mathrm{~Hz}$ with a binning $2 \times 2$ ) exhibit almost the same features. The higher time resolution does not highlight any supplementary 
a)

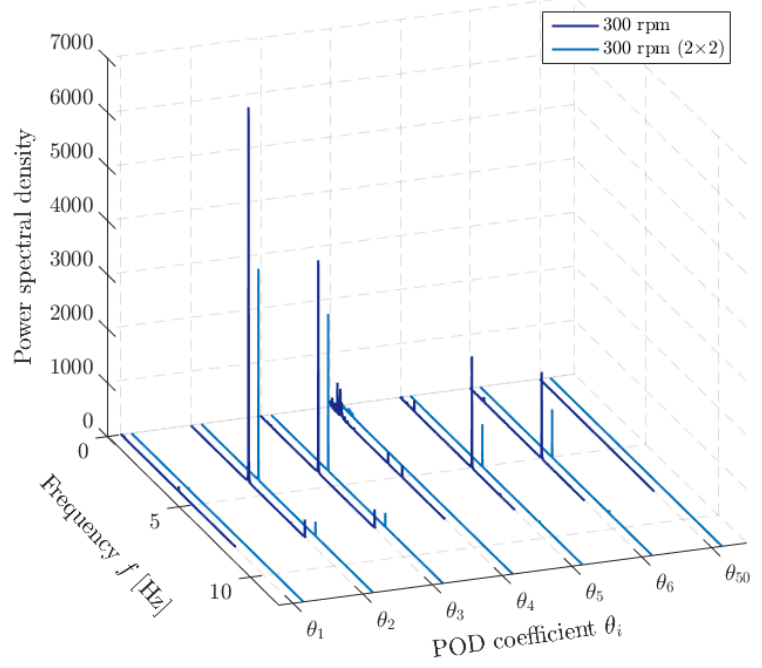

b)

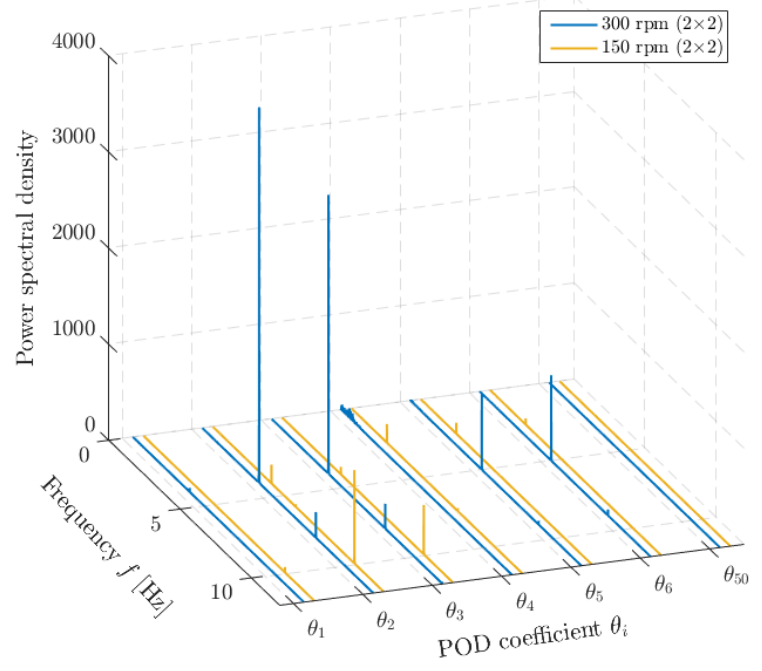

Figure 15: Comparison of the POD coefficients $\theta_{i}(t)$ based on their Lomb-Scargle periodogram $(\mathrm{i}=1,2,3,4,5,6$ and 50$)$ for $N=300 \mathrm{rpm}$ without binning and with binning $2 \times 2$ (a), and for $N=300$ and $150 \mathrm{rpm}$ with binning $2 \times 2$ (b)

frequency content for POD coefficients of these seven modes. As regards the influence of the rotating speed, it can be seen in Fig.15(b) that the POD data obtained for $N=150$ and $300 \mathrm{rpm}$ with binning $2 \times 2$ display similar results. The analysis of the time coefficients in the spectral space reveals the presence of two well-defined frequencies: $f=f_{\mathrm{BPF}}$ for POD modes 2 and 3 (i.e. $10 \mathrm{~Hz}$ for $150 \mathrm{rpm}$ and $4 \mathrm{~Hz}$ for $300 \mathrm{rpm}$ ), and $f=N$ (i.e. $2.5 \mathrm{~Hz}$ for $150 \mathrm{rpm}$ and $5 \mathrm{~Hz}$ for $300 \mathrm{rpm}$ ) for POD modes 5 and 6 . Besides, for $N=150 \mathrm{rpm}$, the power spectral density also reveals peaks at $f=4 \mathrm{~Hz}$ for POD modes 2 and 3, corresponding to the first upper partial of the blade passage frequency. Indeed, the first overtone corresponds to $f=20 \mathrm{~Hz}$ and therefore the frequency of $4 \mathrm{~Hz}$ is its alias.

Consequently, whatever the Reynolds number, the POD modes tend to be similar; their spatial (not shown for $N=150$ rpm) and time characteristics can be interpreted similarly. The dominant frequencies identified in Sect.3.2.1 have been confirmed. The POD modes 2 and 3 are related to the impeller motion and design, while POD modes 5 and 6 are related to the operating conditions only.

The application of POD to the instantaneous velocity fields has highlighted the dominance of particular frequencies associated with the interaction between the blades and the baffles, and with the rotating speed. The DMD technique allows to further investigate these time characteristics of the flow.

The DMD spectra in Fig.16 show the amplitude distribution (a,c,e) and the energy contribution (b,d,f) for different acquisitions parameters $(N=300 \mathrm{rpm}$ acquired without $(\mathrm{a}, \mathrm{b})$ and with binning $2 \times 2(\mathrm{c}, \mathrm{d}))$, and operating conditions $(N=150 \mathrm{rpm}$ with binning $2 \times 2(\mathrm{e}, \mathrm{f}))$.

The main frequencies identified by DMD are in close agreement with the ones given by the POD analysis. The spectra relative to the set obtained at $N=300 \mathrm{rpm}$ with binning reveal that modes associated with $f=9,10$ and $12 \mathrm{~Hz}$ that can be observed on Fig.16(c) are less important in terms of energetic contributions (logarithmic scale) as shown on Fig.16(d). It thus means that the higher time resolution $(24 \mathrm{~Hz}$ instead of $16 \mathrm{~Hz}$ ) does not bring any supplementary information relative to the dynamic behavior of the flow. The dynamic spectra for $N=$ $150 \mathrm{pm}$ with binning present peaks at $f=2.5,4$ and $10 \mathrm{~Hz}$. However, the two lower frequencies are not well evidenced by the energetic contribution spectrum: $f=2.5 \mathrm{~Hz}$ is associated to a small growth rate, and $f=4 \mathrm{~Hz}$ to a low damping rate.

The structures and their spectral properties isolated by the DMD method present strong similarities with those identified by POD. As mentioned above, this concurrence between POD and DMD indeed holds for flow configurations that are dominated by periodic and convective phenomena.

In addition to the trailing vortices generated by the Rushton blades detected at $f=f_{\mathrm{BPF}}$, both modal decomposition methods highlight another coherent structure directly related to the rotating speed, i.e. vortical structures in impeller discharge flow. In the last decades, studies on the flow structures inside stirred-tank driven by Rushton turbine have been focused on the vicinity of the impeller and consequently the trailing vortices (e.g. Riet and Smith (1975), Ranade et al. (2001), Escudié et al. (2004), Delafosse et al. (2009), Liné et al. (2013), Chara et al. (2016)). To the best of knowledge of the authors, the presence of another dominant spatial pattern has never been discussed. The outcomes of the present work have thus: $(i)$ evidenced vortical structures in impeller discharge flow, (ii) detected them at the rotating speed and (iii) thus differentiate them from trailing vortices. However, it is worth noting that these structures are characterized by an energy content much lower than the one associated with the trailing vortices. Consequently, additional investigations should be carried out to explain the origin and occurrence of these flow patterns: for instance, if they are inherent to mechanically agitated tank or if they are related to the agitation configuration (e.g. dual impeller system, high aspect ratio). 
a)

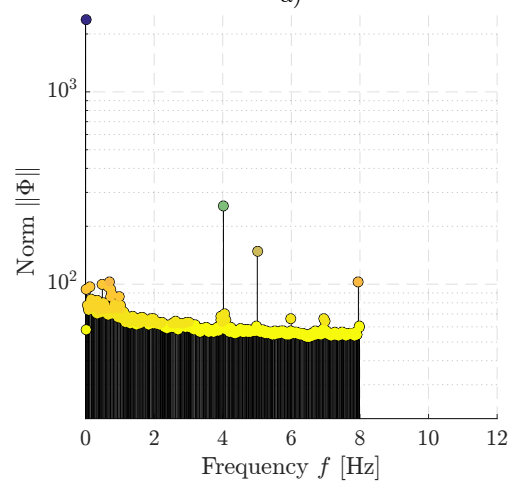

d)

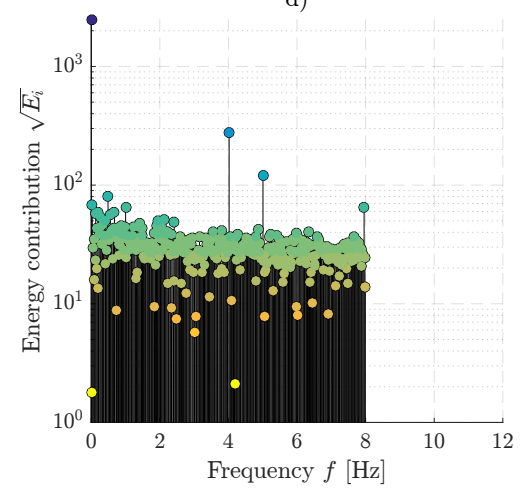

b)

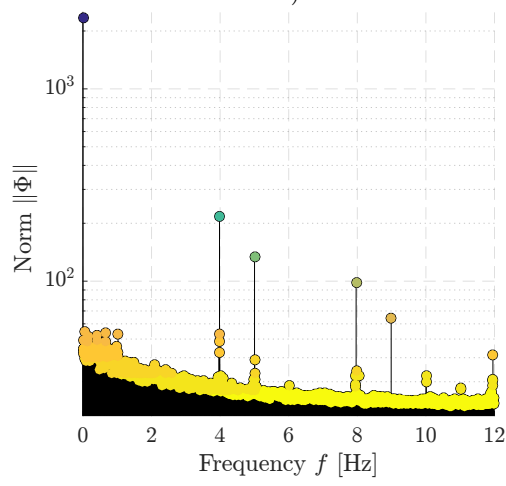

e)

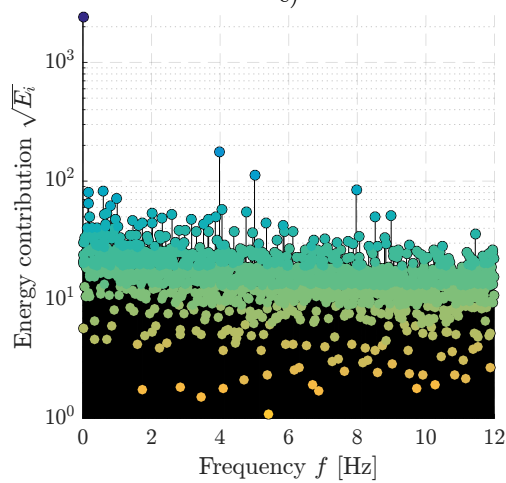

c)

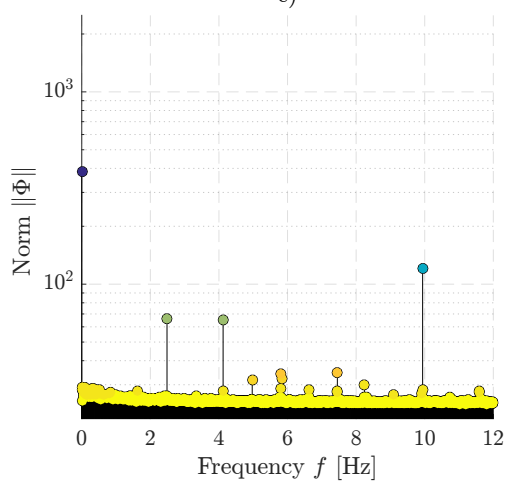

e)

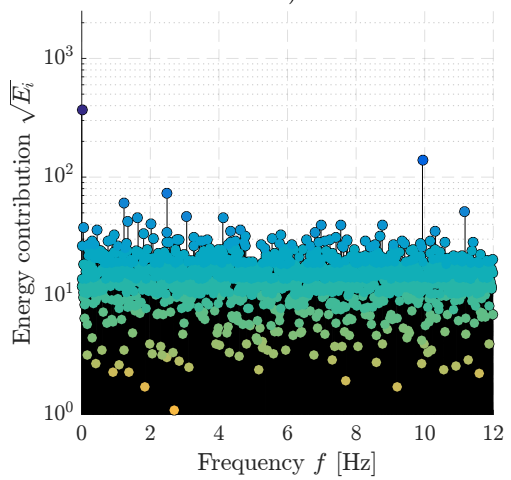

Figure 16: Dynamic mode spectra for $N=300 \mathrm{rpm}$ without binning (a,b) and with binning (c,d) and for $N=150 \mathrm{rpm}$ with binning (e,f): norm $\|\Phi\| v s$. frequency $f$ spectra (a,c,e), energy contribution $\sqrt{E} v s$. frequency $f$ spectra $(\mathrm{b}, \mathrm{d}, \mathrm{f})$

\section{Conclusion}

Data-decomposition methods are used to objectively and quantitatively describe complex flow fields by extracting their most relevant spatial and time features. The POD and DMD techniques have been selected because of their simplicity of application and of their potential complementarity to get a better understanding of unsteady hydrodynamic data and the underlying physical phenomena. POD and DMD analyses have been applied to PIV measurements of instantaneous velocity fields in a turbulent flow generated in a lab-scale vessel stirred by two Rushton impellers at two rotating speeds, $N=150$ and 300 rpm.

As expected, the results show that the flow field is characterized by a wide range of spatial and time scales, but some dominant frequencies may be evidenced clearly.

POD analysis reveals that the first most energetic unsteady structures are related to the trailing vortices induced by the Rushton turbines and are associated to a frequency taken for the blade passage frequency and its overtones,i.e. $f_{\mathrm{BPF}}=10$ and $20 \mathrm{~Hz}$ for $N=150$ and $300 \mathrm{rpm}$. The second most energetic group of modes is related to vortical structures in the impeller jet and are associated to a frequency equivalent to the rotating speed and its overtones, i.e. $f_{N}=2.5$ and $5 \mathrm{~Hz}$ for $N=$ 150 and $300 \mathrm{rpm}$. These frequencies are thus characteristic of the flow structures that are system-dependent, i.e. the agitation configuration and the operating conditions. Moreover, the spatial pattern identified at $f=N$ and characterized by an energy content much lower than the one of the trailing vortices has not been, to the best of knowledge of the authors, reported in previous studies. However, a proper identification requires further investigations on its occurrence and origin.

DMD analysis identifes dynamic modes similar to the most energetic modes evidenced by POD analysis. However, differences appear due to the fact that DMD analysis isolates structures associated with a single frequency only and their corresponding growth/decay rate.

Comparison of the results from the two modal decomposition methods points out their complementarity and their potential to describe the spatial and time characteristics of the flow within a stirred tank. Nevertheless, the DMD technique is superior to POD if one is interested in the flow behavior at certain frequencies (extraction of the spatial structures associated to these dominating frequencies), for example time variations of the mean flow (macro-instabilities) and other instability mechanisms. On the other hand, POD method is more appropriate than DMD if the study is related to the energy transfer between the different characteristic length scales (from the large to the turbulent eddies), for example the rate of total viscous dissipation of kinetic energy and the shear rate.

The present data-processing techniques may lead to many other data and insights on other phenomena such as scalar transport inside stirred-tank reactors by a continued exploration of existing databases or in combination with either new measurements or numerical simulations.

It is of interest to extract the most relevant spatial and time 
features of scalar transfer and transport processes within a stirred tank in order to enhance the understanding of the coupling between flow motions with mixing and gas transfer across the free-surface. The complementarity of the present datadecomposition methods and comparison of their results may thus give access to information on hydrodynamics as well as mixing and/or mass transfer, that is otherwise hard to highlight due to the interactions of several physical mechanisms. With this aim, the energetic dominant flow modes and the spatial structures of the dominating frequencies can then be extracted by applying the POD and DMD techniques to, for instance, PLIF measurements of instantaneous concentration (of an inert tracer or of dissolved gas) fields (de Lamotte et al., 2017).

\section{Acknowledgements}

AdL was supported by a PDR FNRS PhD grant (PDR $\mathrm{n}^{\mathrm{o}}$ T.0250.13) from the National Funds for Scientific Research of Belgium (FRS-FNRS). All the authors gratefully thank T. Salmon and F. Fyon for their technical assistance.

\section{Appendix A. Modal decompositions algorithms}

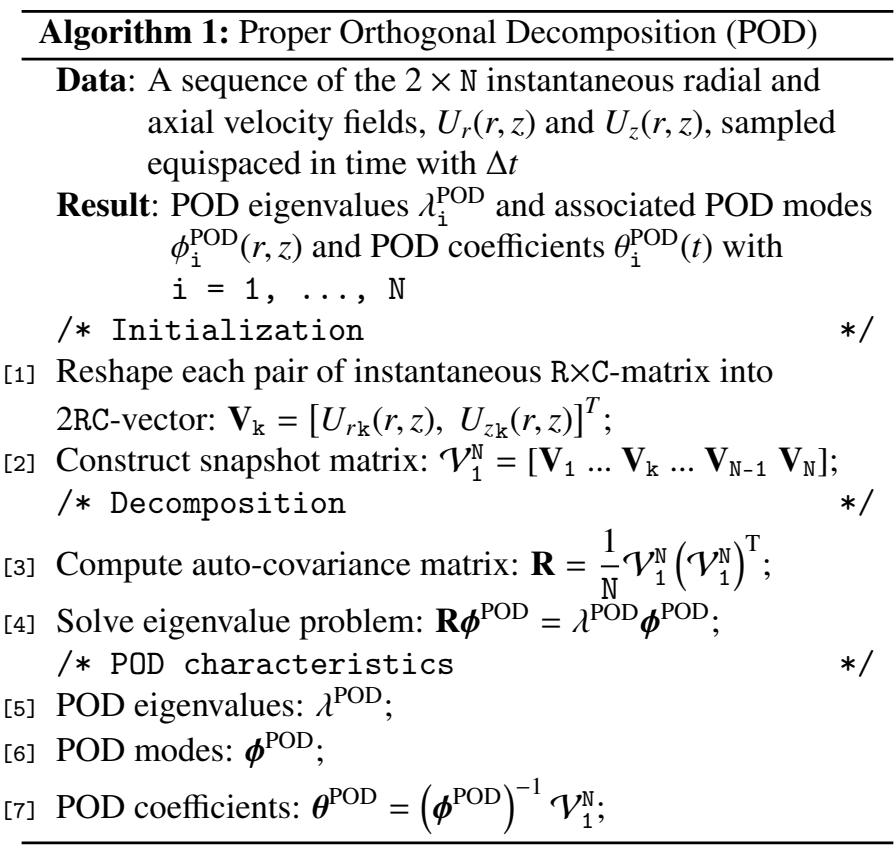

\section{References}

Aubin, J., Sauze, N. L., Bertrand, J., Fletcher, D., Xuereb, C., 2004. Piv measurements of flow in an aerated tank stirred by a down- and an up-pumping axial flow impeller. Experimental Thermal and Fluid Science 28 (5), 447 456, 5th international conference on Gas-Liquid and Gas-Liquid-Solid Reactor Engineering.

Berkooz, G., Holmes, P., Lumley, J. L., 1993. The proper orthogonal decomposition in the analysis of turbulent flows. Annual review of fluid mechanics 25 (1), 539-575.

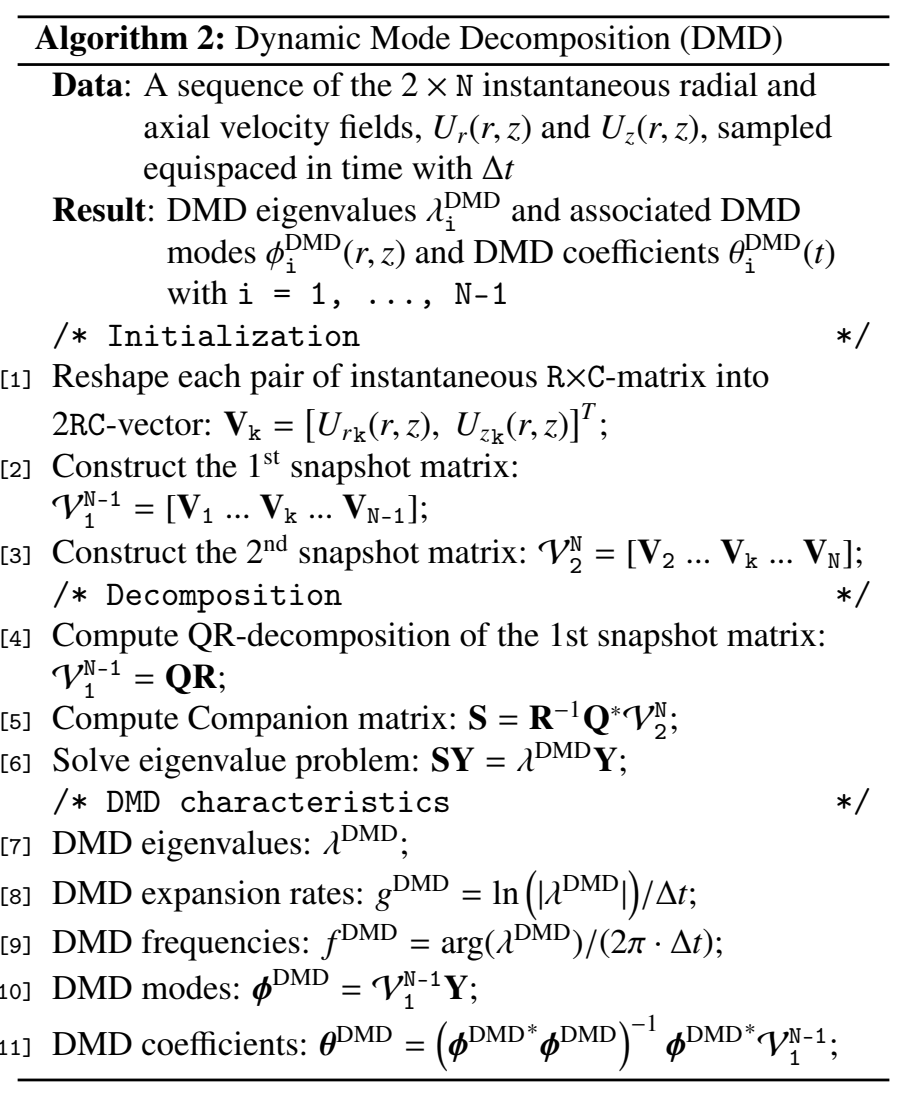

Brucato, A., Ciofalo, M., Grisafi, F., Micale, G., 1998. Numerical prediction of flow fields in baffled stirred vessels: A comparison of alternative modelling approaches. Chemical Engineering Science 53 (21), 3653 - 3684.

Bujalski, W., Nienow, A., Chatwin, S., Cooke, M., 1987. The dependency on scale of power numbers of rushton disc turbines. Chemical Engineering Science 42 (2), $317-326$.

Chara, Z., Kysela, B., Konfrst, J., Fort, I., 2016. Study of fluid flow in baffled vessels stirred by a rushton standard impeller. Applied Mathematics and Computation 272 (Part 3), 614 - 628.

Chatterjee, A., 2000. An introduction to the proper orthogonal decomposition. Current Science 78 (7), 808-817.

Chiti, F., Bakalis, S., Bujalski, W., Barigou, M., Eaglesham, A., Nienow, A. W., 2011. Using positron emission particle tracking (PEPT) to study the turbulent flow in a baffled vessel agitated by a rushton turbine: Improving data treatment and validation. Chemical Engineering Research and Design 89 (10), 1947 - 1960.

de Lamotte, A., Delafosse, A., Calvo, S., Delvigne, F., Toye, D., 2017. Investigating the effects of hydrodynamics and mixing on mass transfer through the free-surface in stirred tank bioreactors. Chemical Engineering Science $172,125-142$.

Deen, N. G., Solberg, T., Hjertager, B. H., 8 2002. Flow generated by an aerated rushton impeller: Two-phase PIV experiments and numerical simulations. The Canadian Journal of Chemical Engineering 80 (4), 1-15.

Deglon, D., Meyer, C., 2006. CFD modelling of stirred tanks: Numerical considerations. Minerals Engineering 19 (10), 1059 - 1068.

Delafosse, A., Collignon, M.-L., Calvo, S., Delvigne, F., Crine, M., Thonart, P., Toye, D., 2014. CFD-based compartment model for description of mixing in bioreactors. Chemical Engineering Science 106, 76 - 85.

Delafosse, A., Collignon, M.-L., Crine, M., Toye, D., 2011. Estimation of the turbulent kinetic energy dissipation rate from 2D-PIV measurements in a vessel stirred by an axial mixel TTP impeller. Chemical Engineering Science 66 (8), 1728 - 1737.

Delafosse, A., Line, A., Morchain, J., Guiraud, P., 2008. Les and urans simulations of hydrodynamics in mixing tank: Comparison to piv experiments. Chemical Engineering Research and Design 86 (12), 1322 - 1330.

Delafosse, A., Morchain, J., Guiraud, P., Liñ̃l', A., 2009. Trailing vortices 
generated by a rushton turbine: Assessment of urans and large eddy simulations. Chemical Engineering Research and Design 87 (4), 401 - 411.

Derksen, J., Doelman, M., Van den Akker, H., 1999. Three-dimensional LDA measurements in the impeller region of a turbulently stirred tank. Experiments in Fluids 27 (6), 522-532.

Ducci, A., Doulgerakis, Z., Yianneskis, M., 2008. Decomposition of flow structures in stirred reactors and implications for mixing enhancement. Industrial \& Engineering Chemistry Research 47 (10), 3664-3676.

Escudié, R., Bouyer, D., Liné, A., 1 2004. Characterization of trailing vortices generated by a rushton turbine. AIChE Journal 50 (1), 75-86.

Escudié, R., Liné, A., 2003. Experimental analysis of hydrodynamics in a radially agitated tank. AIChE Journal 49 (3), 585-603.

Gabriele, A., Nienow, A., Simmons, M., 2009. Use of angle resolved PIV to estimate local specific energy dissipation rates for up- and down-pumping pitched blade agitators in a stirred tank. Chemical Engineering Science 64 (1), $126-143$

Galletti, C., Brunazzi, E., Yianneskis, M., Paglianti, A., 2003. Spectral and wavelet analysis of the flow pattern transition with impeller clearance variations in a stirred vessel. Chemical Engineering Science 58 (17), 3859 3875 .

Guha, D., Dudukovic, M. P., Ramachandran, P. A., Mehta, S., Alvare, J., 5 2006. CFD-based compartmental modeling of single phase stirred-tank reactors. AIChE Journal 52 (5), 1836-1846.

Hasal, P., Fort, I., Kratena, J., 2004. Force effects of the macro-instability of flow pattern on radial baffles in a stirred vessel with pitched-blade and rushton turbine impellers. Chemical Engineering Research and Design 82 (9), $1268-1281$.

Hasal, P., Montes, J.-L., Boisson, H.-C., Fořt, I., 2000. Macro-instabilities of velocity field in stirred vessel: detection and analysis. Chemical Engineering Science 55 (2), $391-401$

Jahoda, M., Moštěk, M., Kukuková, A., Machoš, V., 2007. CFD modelling of liquid homogenization in stirred tanks with one and two impellers using large eddy simulation. Chemical Engineering Research and Design 85 (5), $616-625$

Joshi, J. B., Nere, N. K., Rane, C. V., Murthy, B. N., Mathpati, C. S., Patwardhan, A. W., Ranade, V. V., 2011. CFD simulation of stirred tanks: Comparison of turbulence models. Part I: Radial flow impellers. The Canadian Journal of Chemical Engineering 89 (1), 23-82.

Joshi, J. B., Tabib, M. V., Deshpande, S. S., Mathpati, C. S., 2009. Dynamics of flow structures and transport phenomena, 1. Experimental and numerical techniques for identification and energy content of flow structures. Industrial \& Engineering Chemistry Research 48 (17), 8244-8284.

Liné, A., 2016. Eigenvalue spectrum versus energy density spectrum in a mixing tank. Chemical Engineering Research and Design 108, 13 - 22.

Liné, A., Gabelle, J.-C., Morchain, J., Anne-Archard, D., Augier, F., 2013. On POD analysis of PIV measurements applied to mixing in a stirred vessel with a shear thinning fluid. Chemical Engineering Research and Design 91 (11), 2073 - 2083.

Machado, M. B., Bittorf, K. J., Roussinova, V. T., Kresta, S. M., 2013. Transition from turbulent to transitional flow in the top half of a stirred tank. Chemical Engineering Science 98, 218 - 230.

Mavros, P., 2001. Flow visualization in stirred vessels: A review of experimental techniques. Chemical Engineering Research and Design 79 (2), 113 127

Mezic, I., 2013. Analysis of fluid flows via spectral properties of the koopman operator. Annual Review of Fluid Mechanics 45 (1), 357-378.

Montes, J.-L., Boisson, H.-C., Fořt, I., Jahoda, M., 1997. Velocity field macroinstabilities in an axially agitated mixing vessel. Chemical Engineering Journal 67 (2), 139 - 145.

Moreau, J., Liné, A., 2006. Proper orthogonal decomposition for the study of hydrodynamics in a mixing tank. AIChE Journal 52 (7), 2651-2655.

Raju, R., Balachandar, S., Hill, D., Adrian, R., 2005. Reynolds number scaling of flow in a stirred tank with Rushton turbine. Part II - Eigen decomposition of fluctuation. Chemical Engineering Science 60 (12), 3185 - 3198

Rammohan, A., Kemoun, A., Al-Dahhan, M., Dudukovic, M., 2001. Characterization of single phase flows in stirred tanks via computer automated radioactive particle tracking (CARPT). Chemical Engineering Research and Design 79 (8), 831 - 844.

Ranade, V., Perrard, M., Sauze, N. L., Xuereb, C., Bertrand, J., 2001. Trailing vortices of rushton turbine: PIV measurements and CFD simulations with snapshot approach. Chemical Engineering Research and Design 79 (1), 3 -
12.

Riet, K. V., Smith, J. M., 1975. The trailing vortex system produced by rushton turbine agitators. Chemical Engineering Science 30 (9), 1093 - 1105.

Roussinova, V. T., Kresta, S. M., Weetman, R., 12 2004. Resonant geometries for circulation pattern macroinstabilities in a stirred tank. AIChE Journal 50 (12), 2986-3005.

Rowley, C. W., Mezić, I., Bagheri, S., Schlatter, P., Henningson, D. S., 2009. Spectral analysis of nonlinear flows. Journal of Fluid Mechanics 641, 115127

Ruhe, A., 1984. Rational krylov sequence methods for eigenvalue computation. Linear Algebra and its Applications 58 (Supplement C), 391 - 405.

Rutherford, K., Mahmoudi, S., Lee, K., Yianneskis, M., et al., 1996. The influence of rushton impeller blade and disk thickness on the mixing characteristics of stirred vessels. Chemical engineering research \& design 74 (3), 369-378.

Sakowitz, A., Mihaescu, M., Fuchs, L., 2014. Flow decomposition methods applied to the flow in an IC engine manifold. Applied Thermal Engineering $65(1-2), 57-65$.

Schmid, P. J., 2010. Dynamic mode decomposition of numerical and experimental data. Journal of Fluid Mechanics 656, 5-28.

Schmid, P. J., 2011. Application of the dynamic mode decomposition to experimental data. Experiments in Fluids 50 (4), 1123-1130.

Schmid, P. J., Li, L., Juniper, M. P., Pust, O., Jun 2011. Applications of the dynamic mode decomposition. Theoretical and Computational Fluid Dynamics 25 (1), 249-259.

Schmid, P. J., Violato, D., Scarano, F., 2012. Decomposition of time-resolved tomographic PIV. Experiments in Fluids 52 (6), 1567-1579.

Semeraro, O., Bellani, G., Lundell, F., 2012. Analysis of time-resolved PIV measurements of a confined turbulent jet using POD and koopman modes. Experiments in Fluids 53 (5), 1203-1220.

Sharp, K. V., Adrian, R. J., 2001. PIV study of small-scale flow structure around a rushton turbine. AIChE Journal 47 (4), 766-778.

Sheng, J., Meng, H., Fox, R., 2000. A large eddy piv method for turbulence dissipation rate estimation. Chemical Engineering Science 55 (20), 4423 4434.

Sheng, J., Meng, H., Fox, R. O., 1998. Validation of CFD simulations of a stirred tank using particle image velocimetry data. The Canadian Journal of Chemical Engineering 76 (3), 611-625.

Sirovich, L., 1987. Turbulence and the dynamics of coherent structures. I Coherent structures. Quarterly of Applied Mathematics 45, 561-571.

Tabib, M. V., Joshi, J. B., 2008. Analysis of dominant flow structures and their flow dynamics in chemical process equipment using snapshot proper orthogonal decomposition technique. Chemical Engineering Science 63 (14), 3695 -3715 .

Tissot, G., Cordier, L., Benard, N., Noack, B. R., 2013. Dynamic mode decomposition of PIV measurements for cylinder wake flow in turbulent regime. In: TSFP DIGITAL LIBRARY ONLINE. Begel House Inc.

Tissot, G., Cordier, L., Benard, N., Noack, B. R., 2014. Model reduction using dynamic mode decomposition. Comptes Rendus Mécanique 342 (6-7), 410 -416 .

Tu, J. H., Rowley, C. W., Luchtenburg, D. M., Brunton, S. L., Kutz, J. N., 2014. On dynamic mode decomposition: Theory and applications. Journal of Computational Dynamics 1 (2), 391-421.

Wu, H., Patterson, G., 1989. Laser-doppler measurements of turbulent-flow parameters in a stirred mixer. Chemical Engineering Science 44 (10), 2207 2221.

Zadghaffari, R., Moghaddas, J., Revstedt, J., 2009. A mixing study in a doublerushton stirred tank. Computers \& Chemical Engineering 33 (7), 1240 1246.

Zhang, Q., Liu, Y., Wang, S., 2014. The identification of coherent structures using proper orthogonal decomposition and dynamic mode decomposition. Journal of Fluids and Structures 49, 53 - 72. 